\title{
Complementarity of the CERN LEP collider, the Fermilab Tevatron, and the CERN LHC in the search for a light MSSM Higgs boson
}

\author{
M. Carena* \\ Theory Division, CERN, 1211 Geneva 23, Switzerland \\ S. Mrenna \\ Physics Department, University of California at Davis, Davis, California 95616 \\ C. E. M. Wagner ${ }^{\dagger}$ \\ High Energy Physics Division, Argonne National Laboratory, Argonne, Illinois 60439
}

(Received 1 December 1999; published 1 August 2000)

\begin{abstract}
We study the discovery potential of the CERN LHC, Fermilab Tevatron and CERN LEP colliders in the search for the neutral $C P$-even Higgs boson of the MSSM which couples to the weak gauge bosons with a strength close to the standard model one and, hence, plays a relevant role in the mechanism of electroweak symmetry breaking. We place special emphasis on the radiative effects which influence the discovery reach of these colliders. We concentrate on the $V b \bar{b}$ channel, with $V=Z$ or $W$, and on the channels with diphoton final states, which are the dominant ones for the search for a light standard model Higgs boson at LEP or Tevatron and LHC, respectively. By analyzing the parameters of the MSSM for which the searches become difficult at one or more of these three colliders, we demonstrate their complementarity in the search for a light Higgs boson which plays a relevant role in the mechanism of electroweak symmetry breaking.

PACS number(s): $14.80 . \mathrm{Cp}$
\end{abstract}

\section{INTRODUCTION}

The standard model (SM) of particle physics provides an excellent description of data from collider experiments, including the precision electroweak observables measured at the CERN $e^{+} e^{+}$collider LEP and SLAC Large Detector (SLD). The fit to the data clearly improves if the Higgs boson has a mass less than $250 \mathrm{GeV}$ [1]. LEP is the only accelerator currently running which can directly test for the existence of a standard-model-like Higgs boson, if its mass is sufficiently small [2]. Collaborations using the LEP Collider at CERN have recently performed searches for a standard model Higgs boson at a center of mass energy of $\sqrt{s}=196-202 \mathrm{GeV}$. Preliminary limits on the Higgs boson mass of about $108 \mathrm{GeV}$ were set by the LEP Collaborations [3].

In spite of the phenomenological success of the SM, an explanation of the hierarchy between the Planck and the electroweak scales can only be obtained if new physics is present at scales of the order of the weak scale. The success of the SM in describing the precision electroweak data suggests (although it does not require) that any new physics should be weakly coupled, should lead to small or negligible corrections to precision electroweak observables, and, in addition, should mimic the effects of a light Higgs boson. Low energy supersymmetry provides such an extension of the standard model.

\footnotetext{
* On leave of absence from Fermi National Accelerator Laboratory, Batavia, IL 60510. Email address: carena@fnaligov

${ }^{\dagger}$ On leave of absence from CERN, 1211 Geneva 23, Switzerland. Email address: Carlos.Wagner@cern.ch
}

In the minimal supersymmetric extension of the standard model (MSSM), the Higgs sector contains two doublets. At the tree level, the down and up quarks only couple to the neutral components of the Higgs doublet $H_{1}$ and $H_{2}$, respectively, preventing dangerous flavor-changing neutral current (FCNC) effects. The ratio of the vacuum expectation values of the two Higgs doublets, $v_{1}$ and $v_{2}$, is parametrized by $\tan \beta=v_{2} / v_{1}$. The Higgs spectrum consists of one charged, $H^{ \pm}$, one $C P$-odd, $A$, and two $C P$-even, $h$ and $H$, Higgs bosons. At the tree level all Higgs boson masses may be expressed as functions of $\tan \beta, m_{A}$ and the $W$ and $Z$ boson masses, and an upper bound on the lightest $C P$-even Higgs boson mass is found, $m_{h} \leqslant M_{Z}|\cos 2 \beta|$. This bound is modified by radiative corrections [4], which depend quartically on the top quark mass and logarithmically on the top squark masses [5-9]. As will be discussed below, even after the inclusion of radiative corrections, an upper bound on the lightest $C P$-even Higgs mass is obtained for large values of the $C P$-odd Higgs boson mass $m_{A} \geqslant 300 \mathrm{GeV}$, for which the lightest $C P$-even Higgs boson has standard model-like properties.

The supersymmetric spectrum is constrained by direct experimental searches and by the requirement that it provide a good description of the precision electroweak data. This requirement implies that, unless unnatural cancellations take place [10], the soft supersymmetry-breaking mass parameter for the left-handed top squark should be larger than 300 $\mathrm{GeV}$. Quite generally, the heavier the supersymmetric spectrum, and in particular the heavier the left-handed sfermions, the better the agreement between the MSSM and the precision electroweak observables.

If supersymmetric particles are heavy, the low energy 
properties of the Higgs sector of the MSSM can be described by an effective theory containing two Higgs doublets, with couplings and masses fixed by the proper matching conditions at the scale of the supersymmetric particle masses. In this low energy, effective theory, the couplings of the two $C P$-even Higgs bosons, $h$ and $H$, to the $W$ and $Z$ bosons are given by the SM Higgs couplings multiplied by $\sin (\alpha-\beta)$ and $\cos (\alpha-\beta)$, respectively, where $\alpha$ is the mixing angle of the neutral $C P$-even Higgs bosons. These scaling factors are just the projections of the $C P$-even Higgs bosons on $\Phi$, defined as the combination of the Higgs fields that acquires vacuum expectation value:

$$
\begin{aligned}
\Phi= & \sqrt{2}\left[\operatorname{Re}\left(H_{1}^{0}\right) \cos \beta+\operatorname{Re}\left(H_{2}^{0}\right) \sin \beta\right] \equiv v+h \sin (\beta-\alpha) \\
& +H \cos (\beta-\alpha)
\end{aligned}
$$

where $v \simeq 246 \mathrm{GeV}$ is the SM Higgs vacuum expectation value. In the MSSM, $\Phi$ is not a mass eigenstate. However, $\sin (\alpha-\beta)$ or $\cos (\alpha-\beta)$ becomes close to 1 in large regions of parameter space, reflecting the fact that in those regions it is only one of the two Higgs bosons that is almost entirely responsible for the electroweak symmetry breaking. In particular, for relatively large values of the $C P$-odd Higgs boson mass $\left(m_{A} \geqslant 300 \mathrm{GeV}\right)$, one finds $\sin (\alpha-\beta) \approx 1$.

As shown in the Appendix, there is a useful relation between the masses of the $C P$-even Higgs bosons and their couplings to the $W$ and $Z$ bosons:

$$
m_{h}^{2} \sin ^{2}(\beta-\alpha)+m_{H}^{2} \cos ^{2}(\beta-\alpha)=\left.m_{h}^{2}\right|_{m_{A} \gg M_{Z}} .
$$

In Eq. (1.2), the right hand side is equal to the upper bound on the Higgs boson mass, which, for squark masses of the order of $1 \mathrm{TeV}$, is about $120-130 \mathrm{GeV}$ for moderate or large values of $\tan \beta$ and about $100 \mathrm{GeV}$ for $\tan \beta$ close to one $[7,11]^{1}$

The Higgs boson searches at the LEP Collider, the Fermilab Tevatron and the CERN Large Hadron Collider (LHC) are motivated by the desire to understand the mechanism of electroweak symmetry breaking. The above relation, Eq. (1.2), implies that, if one of the two $C P$-even Higgs bosons couples to the $W$ and $Z$ bosons with a strength close to the SM one $\left[\sin ^{2}(\beta-\alpha)\right.$ of $\cos ^{2}(\beta-\alpha)$ close to 1$]$, it should also be relatively light, with a mass close to the upper bound on the lightest $C P$-even Higgs boson mass for that particular value of $\tan \beta$. Therefore, not only is it true that the lightest $C P$-even Higgs boson mass is bounded from above, but, when $\cos (\beta-\alpha) \approx 1$, then the bound applies to $m_{H}$, with $m_{h}$ being even smaller. If $\sin ^{2}(\beta-\alpha) \simeq \cos ^{2}(\beta-\alpha) \simeq 0.5$, there can be, in principle, a substantial mass splitting between $h$ and $H$, which would imply a light $C P$-even light Higgs $h$. However, as can be seen from Fig. 1, the Higgs boson

\footnotetext{
${ }^{1}$ A similar expression was found in Ref. [12]
}

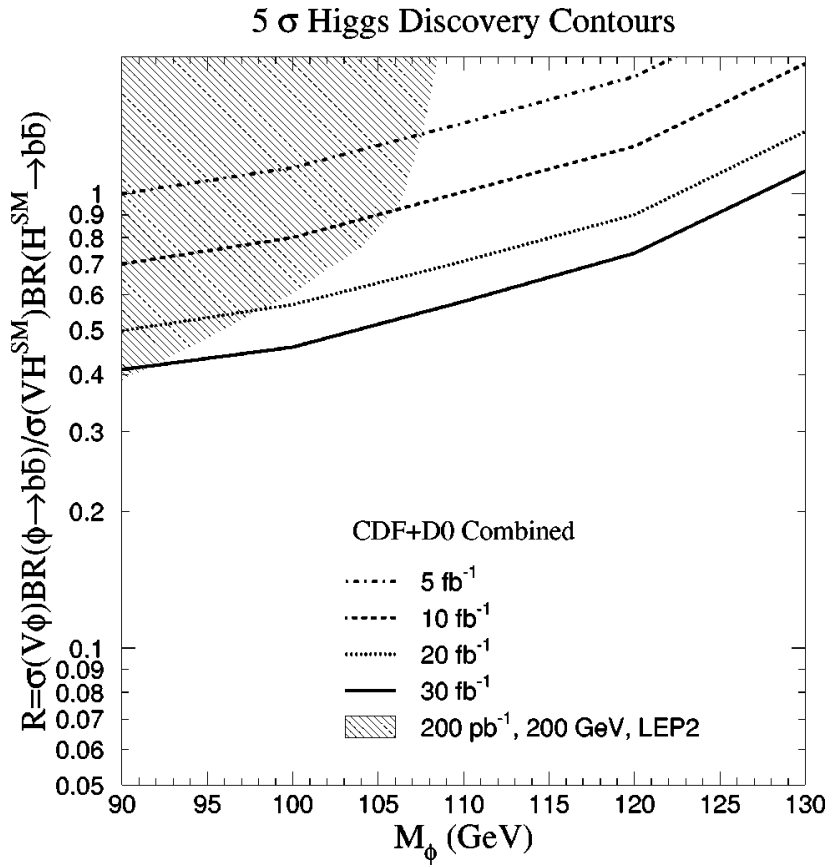

FIG. 1. Sensitivity of the searches at LEP and the Tevatron to a Higgs boson that couples to $W$ and $Z$ bosons and decays to a bottom quark pair. The lines show the minimal value of $R$, Eq. (3.2), necessary to discover a Higgs boson at the Tevatron collider for different values of the total integrated luminosity. At LEP, a Higgs boson can be discovered if, for a given Higgs boson mass, $R$ is within the hatched region.

searches at LEP imply that, under such conditions, $h$ cannot be lighter than $90 \mathrm{GeV}$ and, hence, large mass splittings are experimentally disfavored.

As stated above, in most of the MSSM parameter space one of the two $C P$-even Higgs boson couplings to the weak gauge bosons will be significantly larger than those of the other one and, hence, this Higgs boson will play the most relevant role in the mechanism of electroweak symmetry breaking. In general, we shall denote the $C P$-even Higgs boson with the largest coupling to the weak gauge bosons as $\phi_{W}$, even if the square of this coupling is close to one half of the standard model value. In this work, we shall concentrate on the search for the Higgs $\phi_{W}$ in the MSSM, using only the standard model Higgs boson production channels.

\section{HIGGS BOSON SEARCHES AT PRESENT AND (NEAR) FUTURE COLLIDERS}

There are three colliders where experiments are expected to search for the Higgs $\phi_{W}$ in the mass range $95-130 \mathrm{GeV}$ : the LEP Collider at present, the Tevatron in the years 20012006, and the LHC from 2005 on. As we mentioned above, the collaborations at LEP can search for a Higgs boson with standard model-like couplings to the $Z$ boson and with a mass below or near $105-110 \mathrm{GeV}$ in the channel $Z \phi$ with $\phi \rightarrow b \bar{b}$ or $\tau^{+} \tau^{-}$. If the LEP Collider reaches a center-ofmass energy of about $204 \mathrm{GeV}$ and collects $200 \mathrm{pb}^{-1}$ of data 
per experiment, then evidence for one of the two neutral $C P$-even Higgs bosons will be found by means of the standard model Higgs production channels if:

(i) $\sin ^{2}(\alpha-\beta) \simeq \mathcal{O}(1)\left[\right.$ or $\left.\cos ^{2}(\alpha-\beta) \simeq \mathcal{O}(1)\right]$,

(ii) $C P$-even Higgs mass $m_{h} \lesssim 110 \mathrm{GeV}$ (or $m_{H}$ $\$ 110 \mathrm{GeV}$ ),

(iii) the branching ratio $\mathrm{BR}(h \rightarrow b \bar{b})[$ or $\mathrm{BR}(H \rightarrow b \bar{b})]$ is not much smaller than the standard model value.

The run II of the Tevatron Collider is expected to start in the year 2001. The Tevatron will have sensitivity to Higgs boson in the $V \phi$ channel, with $V=Z$ or $W$, and $\phi \rightarrow b \bar{b}$. Hence, its discovery potential depends also on points number (i) and (iii) above, although the kinematic constraint on the Higgs mass may be relaxed [point (ii)]. However, the Tevatron discovery potential will depend strongly on the final integrated luminosity collected by the Collider Detector at Fermilab (CDF) and D0 experiments [13].

Experiments at the LHC will rely mainly on the signature $p p \rightarrow \gamma \gamma+X$, allowing them to detect the standard model Higgs boson, if its mass is in the range $m_{H} S M \leqslant 130 \mathrm{GeV}$. In particular, the ATLAS and CMS Collaborations have performed studies that show sensitivity to the standard model Higgs boson in the channels $g g \rightarrow \phi(\rightarrow \gamma \gamma), t \bar{t} \phi(\rightarrow \gamma \gamma)$, $W \phi(\rightarrow \gamma \gamma)$, and $t \bar{t} \phi(\rightarrow b \bar{b}){ }^{2}$ These studies, when translated to the search for the Higgs boson $\phi_{W}$ in the MSSM, show that these channels cover wide regions of the $m_{A}-\tan \beta$ plane of the MSSM, with the small $m_{A}$ region $\left(m_{A}\right.$ $\$ 250 \mathrm{GeV}$ ) being the most difficult. Larger coverage of the $m_{A}-\tan \beta$ plane in the MSSM can be achieved by considering also the production and decay signatures of all MSSM Higgs bosons simultaneously $[16,17]$. In these analyses, it is assumed that the sparticles have typical masses ${ }^{3} M_{S}$ of order 1 $\mathrm{TeV}$, and that the top squark trilinear coupling $\widetilde{A}_{t}=A_{t}$ $-\mu / \tan \beta$ is much smaller than $M_{S}$. The latter is, in principle, a conservative assumption, since, for fixed $m_{A}$ and $\tan \beta$, larger values of $\widetilde{A}_{t}$ and $M_{S}$ yield a heavier $\phi_{W}$ which, at low luminosity and for a Higgs boson mass $m_{\phi_{W}}$ $\leqslant 130 \mathrm{GeV}$, is even easier to detect.

The object of the present study is to illustrate the relationship between measurements at the different colliders, and to demonstrate the potential of the combined experimental program to discover the Higgs $\phi_{W}$ boson, by means of the same production channels used for the standard model Higgs boson searches. In the following section, we will review the

\footnotetext{
${ }^{2}$ Several other channels have been proposed which would be useful for studying a Higgs boson with SM-like couplings to the gauge bosons and up quarks $[14,15]$. However, these are experimentally challenging, and, to the best of our knowledge, the ATLAS and CMS Collaborations have not yet analyzed the reach in these channels. We shall therefore not discuss these channels in detail, although we shall analyze their possible relevance in Secs. III and IV C.

${ }^{3}$ To be precise, the supersymmetry (SUSY) scale $M_{S}$ is defined by $M_{S}^{2}=\frac{1}{2}\left(m_{\tilde{t}_{1}}^{2}+m_{\tilde{t}_{2}}^{2}\right)$, where $\tilde{t}_{1}$ and $\tilde{t}_{2}$ are the top squark mass eigenstates.
}

behavior of the Higgs boson couplings to particles and sparticles with respect to variations in the MSSM parameters. In particular, we will show their impact on production cross sections and branching ratios. Given the sensitivity of the various experiments to discover a Higgs boson as a function of the Higgs boson mass and their integrated luminosity, we then calculate the corresponding sensitivity in the MSSM, based on the standard model experimental simulations done for the LEP, Tevatron and LHC colliders, respectively. This will be shown in Sec. IV. We pay particular attention to choices of MSSM parameters which will clearly lead to difficulty at one of the experiments, and explain why this increases the sensitivity of the complementary experiment. Our conclusions are stated in Sec. V.

\section{COUPLINGS OF THE $C P$-EVEN HIGGS BOSONS}

Quite generally, the two $C P$-even Higgs boson eigenstates are mixtures of the real, neutral components of the $H_{1}$ and $\mathrm{H}_{2}$ Higgs doublets,

$$
\left(\begin{array}{l}
h \\
H
\end{array}\right)=\left(\begin{array}{cc}
-\sin \alpha & \cos \alpha \\
\cos \alpha & \sin \alpha
\end{array}\right)\left(\begin{array}{c}
\sqrt{2} \operatorname{Re}\left(H_{1}^{0}\right)-v_{1} \\
\sqrt{2} \operatorname{Re}\left(H_{2}^{0}\right)-v_{2}
\end{array}\right) .
$$

The lightest $C P$-even Higgs boson couples to down quarks and leptons and up quarks with SM Higgs strength times $-\sin \alpha / \cos \beta$ and $\cos \alpha / \sin \beta$, respectively. The corresponding couplings of the heavier $C P$-even Higgs boson are given by the standard model Higgs boson couplings times $\cos \alpha / \cos \beta$ and $\sin \alpha / \sin \beta$, respectively. Analogously, the coupling of the $C P$-odd Higgs boson to down quarks and leptons and up quarks is given by the standard model coupling times $\tan \beta$ and $1 / \tan \beta$, respectively. The lightest [heaviest] $C P$-even Higgs boson has $V V h[V V H]$ couplings which are given by the standard model value times $\sin (\beta$ $-\alpha)[\cos (\beta-\alpha)]$, where $V$ represents a $W$ or $Z$ boson. The coupling of a $C P$-even and a $C P$-odd Higgs boson with a $Z$ boson $Z h A[Z H A]$ is proportional to $\cos (\beta-\alpha)[\sin (\beta$ $-\alpha)]$.

As stated above, LEP is exploring the Higgs boson mass region $m_{\phi_{W}} \lesssim 110 \mathrm{GeV}$. Already the present bounds on a SM-like Higgs boson mass, of about $108 \mathrm{GeV}$, put strong constraints on the realization of the infrared fixed-point scenario in the MSSM and, in general, on the small $\tan \beta$ scenario, with $\tan \beta$ close to one [11]. Indeed, if a SM-like Higgs boson were discovered at LEP in this region of mass, the fixed-point scenario could only be accommodated for large values of the top squark masses and of the top squark mixing parameters. In general, the mass of the Higgs $\phi_{W}$ boson in this range is naturally obtained for $3<\tan \beta \leq 5$. Smaller values of the Higgs boson mass are generically obtained for smaller values of $\tan \beta$. Larger values of the ratio of Higgs vacuum expectation values, $\tan \beta>5$, tend to lead to values of the Higgs $\phi_{W}$ boson mass beyond the reach of LEP. It is also interesting to recall that, in the presence of large mixing in the lepton sector, as suggested by the $\mathrm{Su}$ - 
TABLE I. Tree-level couplings of gauge bosons and bottom and top quarks to the lightest and heaviest $C P$-even Higgs bosons for large values of $\tan \beta$.

\begin{tabular}{lccc}
\hline \hline & $b \bar{b}$ & $t \bar{t}$ & $\mathrm{VV}$ \\
\hline$h$ & $-\sin \alpha \tan \beta$ & $\cos \alpha$ & $\cos \alpha$ \\
$H$ & $\cos \alpha \tan \beta$ & $\sin \alpha$ & $\sin \alpha$ \\
\hline \hline
\end{tabular}

perKamiokande data, these values of $\tan \beta, 3<\tan \beta \leqslant 5$, are consistent with the unification of the bottom and $\tau$ Yukawa couplings at the grand unification scale [18].

As explained above, the present experimental constraints are most naturally satisfied for moderate or large values of $\tan \beta, \tan \beta>3$. For $\tan \beta$ larger than a few, the approximation $\sin \beta \simeq 1$, and hence, $1 / \cos \beta \simeq \tan \beta$, is a good one, and one can construct a table of simplified tree level couplings of fermions and gauge bosons $(V \equiv W$ or $Z$ ) to the $C P$-even Higgs bosons relative to their standard model values given in Table I. Note that the tree level couplings $t \bar{t} \phi$ and $V V \phi$ exhibit the same behavior, so that, for the same values of the Higgs mass the production rates for $Z \phi$ at LEP, $W / Z \phi$ at the Tevatron, and $t \bar{t} / W \phi$ at the LHC are simultaneously enhanced or suppressed with respect to the standard model case. Also, for heavy sparticles, the $\phi \rightarrow g g$ decay rate (which determines the $g g \rightarrow \phi$ production rate) is approximately proportional to the tree level $t \bar{t} \phi$ coupling. Therefore, the production of $t \bar{t} \phi, W \phi$ and $g g \rightarrow \phi$ has the same dependence on the mixing angle $\alpha$ (when the sparticles are heavy). Moreover, when sparticles are heavy, the partial width for the decay of the Higgs boson to a photon pair depends on one-loop contributions from the top quark and $W$ boson, which come with opposite signs. Because of its dependence on the Higgs coupling to the top quark and the $W$ gauge boson, the magnitude of this partial width is also correlated with the ones of the previously mentioned production processes. When the sparticles are light, however, all colorcharged sparticles affect the $\phi g g$ coupling, while all electrically charged sparticles affect the $\phi \gamma \gamma$ coupling, and we will show a few examples in which their effect become relevant. Finally, it is important to remark that, while for $\tan \beta$ larger than a few, the $t \bar{t} \phi$ and $V V \phi$ couplings depend only weakly on $\tan \beta$, the $\phi b \bar{b}$ coupling has a strong dependence on this parameter, and may be strongly affected by radiative corrections proportional to $\tan \beta[19-21,23,24]$. This can have a significant impact on Higgs decay branching ratios.

The LEP experiments are sensitive to the Higgs $\phi_{W}$ mainly through the $Z \phi(\rightarrow b \bar{b})$ process. Given the cross section limit for the standard model Higgs boson, the MSSM limit is derived by properly taking into account the differences between the couplings in the two models. Figure 1 shows the reach of the Higgs boson discovery potential of the LEP and Tevatron colliders for different integrated luminosities of the latter as a function of $R$, defined as the total $V b \bar{b}$ production rate normalized to the standard model value:

$$
R\left(m_{\phi}\right)=\frac{\sigma(V \phi) \mathrm{BR}(\phi \rightarrow b \bar{b})}{\sigma(V \phi)_{S M} \mathrm{BR}(\phi \rightarrow b \bar{b})_{S M}} .
$$

The subscript $S M$ in Eq. (3.2) denotes the standard model values. If $R>1 \quad(R<1)$, then the Higgs boson can be discovered with less (more) luminosity than the standard model one. For the case of the MSSM, the production cross section is only modified by the strength of the $V V \phi$ coupling, so that

$$
R\left(m_{\phi}\right) \simeq\left\{\cos ^{2} \alpha, \sin ^{2} \alpha\right\} \frac{\mathrm{BR}(\phi \rightarrow b \bar{b})}{\mathrm{BR}(\phi \rightarrow b \bar{b})_{S M}}
$$

where the left (right) portion of the expression in brackets refers to $\phi=h(H)$, and we have used the approximations of Table I. If $R$ is too small, then discovery of a SM-like Higgs boson will not be possible. Problematic regions are when (a) $h \equiv \phi_{W}$ or $H \equiv \phi_{W}$ but $m_{h}$ or $m_{H}$ is too large to be kinematically accessible, (b) $h(H)$ has SM-like couplings to the gauge bosons, but $\operatorname{BR}\left(\phi_{W} \rightarrow b \bar{b}\right)$ is suppressed either because the mixing angle $\alpha$ is such that $\sin \alpha / \cos \beta$ $\ll 1(\cos \alpha / \cos \beta \ll 1)$ or because large radiative corrections, induced by supersymmetric particles, cancel its tree level part, leading to a suppression of the renormalized $\phi_{W} b \bar{b}$ coupling, and (c) $\cos ^{2}(\beta-\alpha) \simeq \sin ^{2}(\beta-\alpha)$ so that the production cross section for each of the Higgs bosons is one half of that expected for a standard model Higgs boson of the corresponding Higgs mass, and $m_{h}$ and $m_{H}$ are sufficiently different in mass, so that their decay signatures cannot be combined in the experimental analysis. ${ }^{4}$ The experiments in run II and run III (the proposed high-luminosity runs) at the Tevatron are sensitive to both $W \phi(\rightarrow b \bar{b})$ and $Z \phi(\rightarrow b \bar{b})$ processes. Since these production processes depend on the same couplings, the previous discussion holds both for the LEP and the Tevatron colliders, except that at the Tevatron (a) does not occur, although, as shown in Fig. 1, the discovery reach is strongly dependent on the total integrated luminosity received by the experiments.

As stated above, the $\phi_{W} b \bar{b}$ coupling can become small because a tree level coupling vanishes or because of large radiative corrections. The former occurs when $\sin \alpha$ or $\cos \alpha$ vanishes, whereas, as explained below, the latter holds for special combinations of the soft supersymmetry-breaking parameters, but tends to occur for small values of $\sin \alpha$ or $\cos \alpha$, such that the tree level coupling is non-vanishing, but suppressed compared to the SM one. The value of $\alpha$ is determined by diagonalizing the quadratic mass matrix $\mathcal{M}^{2}$ for the $C P$-even Higgs bosons. Analytic expressions for the Higgs boson squared mass matrix elements and definitions of our notation relevant for our discussion can be found in Refs. $[7,24]$. Here, we correct a minor error in the expression for $\mathcal{M}_{12}^{2}$ in Ref. [24]:

\footnotetext{
${ }^{4} \mathrm{~A}$ reduction of the production cross section by a factor of $1 / 2$ implies that the luminosity should be increased by approximately a factor of 4 , in order to achieve the same discovery reach.
} 


$$
\begin{aligned}
\mathcal{M}_{12}^{2} \simeq & -\left[m_{A}^{2}+M_{Z}^{2}-\frac{h_{t}^{4} v^{2}}{8 \pi^{2}}\left(3 \bar{\mu}^{2}-\bar{\mu}^{2} \bar{A}_{t}^{2}\right)\right] \sin \beta \cos \beta \\
& +\left[\frac{h_{t}^{4} v^{2}}{16 \pi^{2}} \sin ^{2} \beta \bar{\mu} \bar{A}_{t}\left[\bar{A}_{t}^{2}-6\right]+\frac{3 h_{t}^{2} M_{Z}^{2}}{32 \pi^{2}} \bar{\mu} \tilde{a}\right] \\
& \times\left[1+\frac{t}{16 \pi^{2}}\left(4.5 h_{t}^{2}-0.5 h_{b}^{2}-16 g_{3}^{2}\right)\right] \\
& +\frac{h_{b}^{4} v^{2}}{16 \pi^{2}} \sin ^{2} \beta \bar{\mu}^{3} \bar{A}_{b} \\
& \times\left[1+\frac{t}{16 \pi^{2}}\left(7.5 h_{b}^{2}-3.5 h_{t}^{2}-16 g_{3}^{2}\right)\right] .
\end{aligned}
$$

The final, numerical results of our analysis make use of the complete one-loop renormalization group (RG) improved effective potential computation [7] of the Higgs boson squared mass matrix elements, which allows a more reliable treatment of the cases in which the squark mixing terms or the squark mass splittings become large.

The approximation that $\sin \beta \simeq 1$, which is good for moderate or large values of $\tan \beta$, is equivalent to the relation $v_{2}^{2} \gg v_{1}^{2}$. In this limit, $H_{2}$ is the Higgs doublet mainly responsible for electroweak symmetry breaking and the mass of the Higgs $\phi_{W}$ boson is well approximated by $\sqrt{\mathcal{M}_{22}^{2}}$ (see Ref. [24]), where the $m_{A}$ dependence is suppressed by the large $\tan \beta$ factor. On the other hand, in the limit that $\mathcal{M}_{12} \rightarrow 0$, either $\sin \alpha$ or $\cos \alpha \rightarrow 0$. In the case that $\mathcal{M}_{11}^{2}>\mathcal{M}_{22}^{2}$, it is $\sin \alpha$ that vanishes. Otherwise, it is $\cos \alpha$ that vanishes. Because $\mathcal{M}_{11}^{2} \simeq m_{A}^{2} \sin ^{2} \beta \simeq m_{A}^{2}$, it is $\sin \alpha$ that is suppressed when the off-diagonal elements of the quadratic mass matrix are small and $m_{A}$ is large. In both cases it is $\operatorname{BR}\left(\phi_{W} \rightarrow b \bar{b}\right)$ that is suppressed. Observe that the tree-level contribution to the Higgs boson mass matrix element $\mathcal{M}_{12}^{2}$ is suppressed by a $1 / \tan \beta$ factor. This factor does not lead in general to a suppression of the effective $\phi_{W}$ coupling to bottom quarks, but compensates the $\tan \beta$ enhancement of the bottom Yukawa coupling $h_{b}$ to render it standard model like (see Table I). What is emphasized above is an additional suppression, which only takes place when $\mathcal{M}_{12}^{2}$ is significantly smaller than the tree level value. In general, the radiative corrections are very important and depend on the sign and size of $\bar{\mu} \times \bar{A}_{t}$ and $\bar{\mu} \times \bar{A}_{b}$. The possibility of such effects will not be apparent if one assumes $\mu \simeq 0$ or $A_{t}, A_{b} \simeq 0$.

Up to this point, the discussion has made use of the treelevel (but QCD corrected) relation between the Yukawa couplings and the quark masses. However, for large values of $\tan \beta$, there can be a significant modification of the bottom and possibly tau Yukawa couplings from SUSY corrections [19-21,23-25]. For completeness, we provide the modifications to the $\phi b \bar{b}$ couplings derived by us earlier in an effective Lagrangian approach [24]. The starting point is the effective Lagrangian at energies below the supersymmetric particle masses, which are assumed to be larger than the weak scale, $M_{S}^{2} \gg M_{Z}^{2}$ :

$$
\mathcal{L} \simeq h_{b} H_{1}^{0} b \bar{b}+\Delta h_{b} H_{2}^{0} b \bar{b} .
$$

In the above, the appearance of the one-loop generated coupling $\Delta h_{b}$ is a reflection of the breakdown of supersymmetry at low energies. The lightest $C P$-even Higgs boson coupling to bottom quarks $h_{b, h}$ is approximately given by ${ }^{5}[24,25]$

$$
h_{b, h} \simeq-\frac{m_{b} \sin \alpha}{v \cos \beta}\left[1-\frac{\Delta\left(m_{b}\right)}{1+\Delta\left(m_{b}\right)}\left(1+\frac{1}{\tan \alpha \tan \beta}\right)\right],
$$

and a similar expression holds for the heaviest $C P$-even Higgs boson with the interchange $\sin \alpha \rightarrow-\cos \alpha, \tan \alpha \rightarrow$ $-1 / \tan \alpha$. The function $\Delta\left(m_{b}\right)=\left(\Delta h_{b} / h_{b}\right) \tan \beta$ contains two main contributions, one from a bottom-squark-gluino loop (depending on the two bottom squark masses $M_{\tilde{b}_{1}}$ and $M_{\tilde{b}_{2}}$ and the gluino mass $M_{\tilde{g}}$ ) and another one from a topsquark-Higgsino loop (depending on the two top squark masses $M_{\tilde{t}_{1}}$ and $M_{\tilde{t}_{2}}$ and the Higgsino mass parameter $\mu$ ). The functional form of $\Delta\left(m_{b}\right)$, calculated approximately at one loop and at zero external momentum $\left(M_{S} \gg m_{b}\right)$, can be found in Refs. [19-22]. The value of $\Delta\left(m_{b}\right)$ is defined at the scale $M_{S}$, where the sparticles are decoupled. The $h_{b}$ and $\Delta h_{b}$ couplings should be computed at that scale and run down with their respective renormalization group equations to the scale $m_{A}$, where the relations between the couplings of the bottom quark to the neutral Higgs bosons and the running bottom quark mass are defined.

The $C P$-even Higgs boson couplings to the $\tau$ leptons are also affected by large corrections at large $\tan \beta$. They are given by similar expressions as the ones for the bottom couplings, but replacing $\Delta\left(m_{b}\right)$ by $\Delta\left(m_{\tau}\right)[24,25]$, with $\Delta\left(m_{\tau}\right)$ proportional to weak gauge couplings and, therefore, usually much smaller than $\Delta\left(m_{b}\right)$ [21].

In the earlier discussion of the suppression of the $\phi_{W} b \bar{b}$ coupling, an implicit assumption was made that $\Delta\left(m_{b}\right)$ and $\Delta\left(m_{\tau}\right)$ were small. In the limit $\sin \alpha=0(\cos \alpha=0)$, the $\phi b \bar{b}$ coupling is given by

$$
h_{b, h}\left(h_{b, H}\right)=\frac{m_{b}}{\sin \beta v} \frac{\Delta\left(m_{b}\right)}{1+\Delta\left(m_{b}\right)} \equiv \Delta h_{b},
$$

which is very small if $\left|\Delta\left(m_{b}\right)\right| \ll 1$. A similar expression to Eq. (3.7) holds for the $\tau$ lepton coupling.

The bottom mass correction factor $\Delta\left(m_{b}\right) \ll 1$, except when $\tan \beta$ and/or the top squark mixing mass parameters $A_{t}$ and $\mu$ are large, in which case it can be near unity. When $\Delta\left(m_{b}\right)$ is of order 1 , the $\phi_{W} b \bar{b}$ coupling can be of the order of the standard model one even though $\sin \alpha \cos \alpha \rightarrow 0$. Moreover, since $\Delta\left(m_{b}\right)$ and $\Delta\left(m_{\tau}\right)$ will be different in general, their relative strength can be quite different from that in the SM or the tree-level MSSM. It is possible for $h_{\tau, h}$ to vanish, while $h_{b, h}$ is substantial $[24,25]$. Note that a strong suppres-

\footnotetext{
${ }^{5}$ These results agree with those obtained by diagrammatic computations $[26,27]$.
} 
sion of the bottom coupling $h_{b, h}$ can still occur for slightly different values of the Higgs mixing angle $\alpha$, namely

$$
\tan \alpha \simeq \frac{\Delta\left(m_{b}\right)}{\tan \beta} \equiv \frac{\Delta h_{b}}{h_{b}} .
$$

Under these conditions,

$$
h_{\tau, h}=\frac{m_{\tau}}{v \sin \beta}\left(\frac{\Delta\left(m_{\tau}\right)-\Delta\left(m_{b}\right)}{1+\Delta\left(m_{\tau}\right)}\right), \quad h_{b, h}=0 .
$$

A similar expression is obtained for the coupling $h_{\tau, H}$ in the case $h_{b, H}=0$. Hence, if $\tan \beta$ is very large and $\Delta\left(m_{b}\right)$ is of order 1 , the $\tau$ Yukawa coupling may not be strongly suppressed with respect to the standard model case and can provide the dominant decay mode for a standard model-like Higgs boson. Likewise, a suppression of the $h_{\tau, h}$ coupling arises for $\tan \alpha \simeq \Delta\left(m_{\tau}\right) / \tan \beta$.

At the tree level, when $\phi_{W} \rightarrow b \bar{b}$ vanishes, so does $\phi_{W}$ $\rightarrow \tau^{+} \tau^{-}$. Therefore, the Higgs boson decays to $g g, c \bar{c}, W^{*} W^{*}$ and $\gamma \gamma$ occur at enhanced rates compared to the SM expectations. Once the vertex corrections are included, the results will depend on $\Delta\left(m_{b}\right)$ [we assume for the rest of this discussion that $\Delta\left(m_{\tau}\right)$ is small]. Since the $\Delta\left(m_{b}\right)$ corrections depend strongly on the size and sign of $M_{\tilde{g}}$, and hence introduce a dependence on parameters which do not otherwise affect the Higgs boson masses and mixing angles in a significant way, we shall neglect them in the main analysis. However, in Sec. IV, we shall present a dedicated analysis of the possible effects of these corrections on the Higgs phenomenology.

The vanishing of the $\phi_{W} b \bar{b}$ coupling may render the Higgs boson searches at LEP and the Tevatron problematic. The enhanced decays $\phi_{W} \rightarrow g g$ and $\phi_{W} \rightarrow c \bar{c}$ are difficult to observe at the LEP and particularly at the Tevatron collider because of increased backgrounds. The "trilepton" signature from $W \phi_{W}\left(\rightarrow W^{*} W^{*}\right)$ may be challenging at the Tevatron [28], because of the small signal rate. While the cross section for the process $g g \rightarrow \phi_{W} \rightarrow \gamma \gamma$ can be enhanced up to about $10 \mathrm{fb}$ at the Tevatron collider, which may be observable, a detailed study of the $\gamma \gamma$ backgrounds in the mass range around $100 \mathrm{GeV}$ is still lacking.

At the LHC, search strategies in the $\gamma \gamma+X$ final state change from the low luminosity run (collecting up to $30 \mathrm{fb}^{-1}$ ) to the high luminosity run $\left(30-100 \mathrm{fb}^{-1}\right.$ and up to $300 \mathrm{fb}^{-1}$ ) because of the relative behavior of the signals and backgrounds. At low luminosity, the experiments are most sensitive to the subprocess $g g \rightarrow \phi_{W} \rightarrow \gamma \gamma$. Given the reach for a SM Higgs boson, the reach in the MSSM can be calculated using the factor $R^{\prime}\left(m_{\phi}\right)$ :

$$
R^{\prime}\left(m_{\phi}\right)=\frac{\Gamma(\phi \rightarrow g g) \operatorname{BR}(\phi \rightarrow \gamma \gamma)}{\Gamma(\phi \rightarrow g g)_{S M} \operatorname{BR}(\phi \rightarrow \gamma \gamma)_{S M}} .
$$

At high luminosity, the best reach for a Higgs boson with SM-like couplings to the gauge bosons is in the $W \phi_{W}(\rightarrow \gamma \gamma)$ and $t \bar{t} \phi_{W}(\rightarrow \gamma \gamma)$ channels. In this case, the production cross section depends on a tree level coupling, and loop effects arise only in $\operatorname{BR}\left(\phi_{W} \rightarrow \gamma \gamma\right)$. The relevant factor $R^{\prime \prime}\left(m_{\phi}\right)$ is

$$
R^{\prime \prime}\left(m_{\phi}\right) \simeq\left\{\cos ^{2} \alpha, \sin ^{2} \alpha\right\} \frac{\mathrm{BR}(\phi \rightarrow \gamma \gamma)}{\operatorname{BR}(\phi \rightarrow \gamma \gamma)_{S M}} .
$$

Let us analyze the properties of the Higgs sector relevant to $R^{\prime}$ and $R^{\prime \prime}$ in more detail. The effect of a light top squark (or light bottom squark at large $\tan \beta)$ with $\widetilde{A}_{t}\left(\widetilde{A}_{b} \equiv A_{b}\right.$ $-\mu \tan \beta$ ) large is to partially cancel the top quark contribution to the loop induced amplitudes of $\phi_{W} \rightarrow \gamma \gamma(\mathrm{gg})$ processes. This decreases the partial width $\Gamma\left(\phi_{W} \rightarrow g g\right)$, but increases $\operatorname{BR}\left(\phi_{W} \rightarrow \gamma \gamma\right)$ since in the standard model the $W$ and $t$ contributions destructively interfere, with the former being the dominant one. For large values of $\tan \beta$, the bottom squarks couple to $\phi_{W}$ with a strength proportional to $m_{b} \tan \beta$ and, if they are sufficiently light, can give a substantial contribution to the width $\Gamma\left(\phi_{W} \rightarrow \gamma \gamma(g g)\right)$, of the same sign as the top squark one. Hence, for sufficiently light third generation squarks, the factor $R^{\prime}$, defined in Eq. (3.10) can be significantly decreased [29] because of a cancellation between the top quark loops and the top and bottom squark loops. (Of course, the presence of light sparticles implies that the next generation of experiments can directly probe them.) The advantage of the $t \bar{t} \phi_{W}$ channel is that $R^{\prime \prime}$ depends on $\operatorname{BR}\left(\phi_{W}\right.$ $\rightarrow \gamma \gamma)$ and not on $\Gamma\left(\phi_{W} \rightarrow g g\right)$. Therefore, the decrease in $R^{\prime}$ can be compensated by an increase of $R^{\prime \prime}$.

Another way to modify $R^{\prime}$ and $R^{\prime \prime}$ is to change one of the tree level couplings listed above. For instance, when $\tan \beta$ is large and $\sin \alpha$ is small, the lightest $C P$-even Higgs boson has standard model-like couplings to the $W$ and $Z$ gauge bosons $\left(h \equiv \phi_{W}\right)$. However, the $\phi_{W}$ coupling is proportional to $\sin \alpha \tan \beta$, and even when $\sin \alpha$ is small, its value depends on the exact characteristics of the supersymmetric spectrum. When $\sin \alpha \tan \beta$ is larger than 1 , the $\operatorname{BR}\left(\phi_{W} \rightarrow b \bar{b}\right)$ becomes larger than the SM one, and, since it is the dominant Higgs boson decay channel, it partially suppresses the $\operatorname{BR}\left(\phi_{W}\right.$ $\rightarrow \gamma \gamma)$. On the contrary, if $\operatorname{BR}\left(\phi_{W} \rightarrow b \bar{b}\right)$ becomes much smaller than 1 , something that, as we explained above, can happen in certain regions of parameters [24], the $\operatorname{BR}\left(\phi_{W}\right.$ $\rightarrow \gamma \gamma$ ) will be strongly enhanced, improving the LHC prospects of finding a light $C P$-even Higgs boson without going to the highest luminosity runs.

Our discussion of searches for a SM-like Higgs boson at the LHC is limited to those presented by the experiments themselves. ATLAS has presented encouraging numbers for their reach in the $t \bar{t} \phi(\rightarrow b \bar{b})$ channel. $^{6}$ In the approximation of Table I, we observe that this process is enhanced (suppressed) in the same regions of MSSM parameters space in which $V \phi(\rightarrow b \bar{b})$ at the LEP and Tevatron colliders is also enhanced (suppressed). This potentially important channel is not included in our analysis. Let us mention, however, that, if the Higgs boson were discovered at LEP and/or the Teva-

\footnotetext{
${ }^{6}$ At low luminosity, the process $W \phi(\rightarrow b \bar{b})$ can also be used.
} 
tron, this channel will serve to explore its coupling to the top quark, providing an important independent test of the origin of fermion masses. On the other hand, if the effective luminosity at the next run of the Tevatron were small, this channel will provide additional means for the LHC to test the Higgs boson responsible for electroweak symmetry breaking in most of the MSSM parameter space. Hence, this Higgs boson production channel at LHC adds to the complementary physics potential of the three colliders that we are emphasizing in this article.

\section{RESULTS}

For our analysis, we rely on the projected discovery reach of the three colliders for a standard model Higgs boson. For LEP2, running at $\sqrt{s}=200 \mathrm{GeV}$ and collecting $200 \mathrm{pb}^{-1}$ of data (per experiment), we use the numbers of Ref. [30]. For the Tevatron in run II and run III, we use the results of the Higgs Working Group of the Workshop on Physics at run II [13]. For the LHC, we use the technical design reports and updates of the ATLAS [16] and CMS [17] Collaborations. A comparison of the sensitivity of the two experiments in the $\gamma \gamma$ channels reveals that CMS is substantially more sensitive than ATLAS. Therefore, only the projected reach of the CMS detector is explicitly used in our analysis to represent the reach of the LHC. To demonstrate the potential of the LHC if $300 \mathrm{fb}^{-1}$ of data is collected, we scale the $100 \mathrm{fb}^{-1}$ significances by a factor of $\sqrt{3}$. The significance in the MSSM is determined by rescaling the partial widths and branching ratios accordingly.

As discussed in Sec. III, some choices for the softsupersymmetry-breaking parameters at the weak scale can make it difficult to observe the Higgs $\phi_{W}$ boson at LEP and the Tevatron in the $\phi_{W} \rightarrow b \bar{b}$ channel or at LHC in the $\phi_{W}$ $\rightarrow \gamma \gamma$ channel. In this section, we demonstrate that, quite generally, difficulties will not arise in both channels simultaneously, provided that the experiments operate efficiently and receive enough integrated luminosity.

We construct our argument by concentrating on MSSM parameters that are problematic for one of the channels and then displaying the complementarity of the other channel. Our choices include the possibility of light top and bottom squarks, whose one-loop contributions can efficiently suppress some of the decay modes of $\phi_{W}$. Higgs boson properties are calculated using HDECAY [31]. However, because of the relevance of the bottom quark mass corrections in defining the proper bottom quark Yukawa coupling at the scale $M_{S}$, we have modified the effective potential calculation of Ref. [7], used in HDECAY, in order to include these corrections in the large $\tan \beta$ regime. The inclusion of these corrections in the expressions for the Higgs boson masses leads to non-leading logarithmic two-loop corrections. Although in a complete two-loop calculation there might be other nonlogarithmic terms modifying the Higgs boson masses, these corrections are particularly important, since they can modify the one-loop result by factors of order 1 .

\section{A. Minimal mixing model}

For large values of $M_{A}$, and for a given choice of $\mu$ and top squark masses, the case $\widetilde{A}_{t}=0$ yields the smallest value of the lightest $C P$-even Higgs boson mass for any choice of $\tan \beta$ (large values of $\tan \beta$, small bottom squark masses or large values of $\mu$ tend to lower the $M_{h}$ values even further [7]). We fix the overall scale of supersymmetry particle masses $M_{S}=1 \mathrm{TeV}$. Furthermore, for comparison with the results presented by ATLAS and CMS, we choose $\mu=$ $-100 \mathrm{GeV}$, which is small enough that it does not differ significantly from the $\mu=0$ case, but large enough to avoid a light chargino. In our scan of the $m_{A}-\tan \beta$ plane, we tune $A_{t}=\mu / \tan \beta$ to achieve the conditions of minimal mixing at each point in the plane. This choice of parameters leads to a moderate value for the lightest $C P$-even Higgs boson mass at large values of $M_{A}$ and $\tan \beta, M_{h} \simeq 115 \mathrm{GeV}$, which is, however, beyond the kinematic reach of LEP. This is a conservative assumption for the LHC experiments, since the discovery reach is improved for larger values of $A_{t}$, which lead to larger values of the Higgs $\phi_{W}$ boson mass.

The regions of the $M_{A}$-tan $\beta$ plane in which a Higgs $\phi_{W}$ boson would be discovered at the $5 \sigma$ level at the Tevatron are shown as shaded regions in Fig. 2(a). Different shadings correspond to different assumptions about the luminosity delivered to both experiments. The LEP discovery contour (for a Higgs $\phi_{W}$ boson) is shown by the double line (the region below the contour will be probed). With more than $5 \mathrm{fb}^{-1}$, the Tevatron begins to provide information beyond that from LEP, provided that the Higgs boson is not already discovered. Up to $30 \mathrm{fb}^{-1}$ of data is needed to cover the problematic region around $\sin ^{2}(\beta-\alpha) \simeq \cos ^{2}(\beta-\alpha)$ at low $\tan \beta$ where $m_{h}$ and $m_{H}$ are separated by more than $\simeq 10 \mathrm{GeV}$ $[32,24]$.

In Fig. 2(b), the corresponding discovery reach with the CMS experiment is shown. The complementarity of the colliders is clear. The LHC experiments are most sensitive to large $m_{A}$, where $m_{h}$ approaches its upper bound for the given choice of parameters. As $m_{A}$ decreases, the $\phi_{W} b \bar{b}$ coupling increases, so that $B\left(\phi_{W} \rightarrow \gamma \gamma\right)$ and $R^{\prime}$ and $R^{\prime \prime}$ decrease. At the Tevatron, the region of large $m_{A}$ and large $\tan \beta$ is harder to cover, because the $\phi_{W} b \bar{b}$ coupling decreases towards the standard model value, yielding $R \simeq 1$. Simultaneously, the lightest $C P$-even Higgs boson mass approaches its upper bound, so that more integrated luminosity is needed to probe this region. It is worth noting the change in the shape of the LHC contours between low and high luminosity, when the $g g \rightarrow \phi_{W}$ process becomes less important than the $t \bar{t} \phi_{W} / W \phi_{W}$ process. After the low luminosity run at the LHC $\left(30 \mathrm{fb}^{-1}\right)$, if the Tevatron obtains only 10 $\mathrm{fb}^{-1}$ of data, there remains a region uncovered by both colliders for $m_{A} \simeq 250-300 \mathrm{GeV}$ [there would also still be the hole at $\left.\sin ^{2}(\beta-\alpha) \simeq \cos ^{2}(\beta-\alpha)\right]$. The high luminosity run (but only with $100 \mathrm{fb}^{-1}$ ) is necessary to guarantee full coverage in this region, unless the Tevatron collects $20 \mathrm{fb}^{-1}$ or more of integrated luminosity per experiment. 

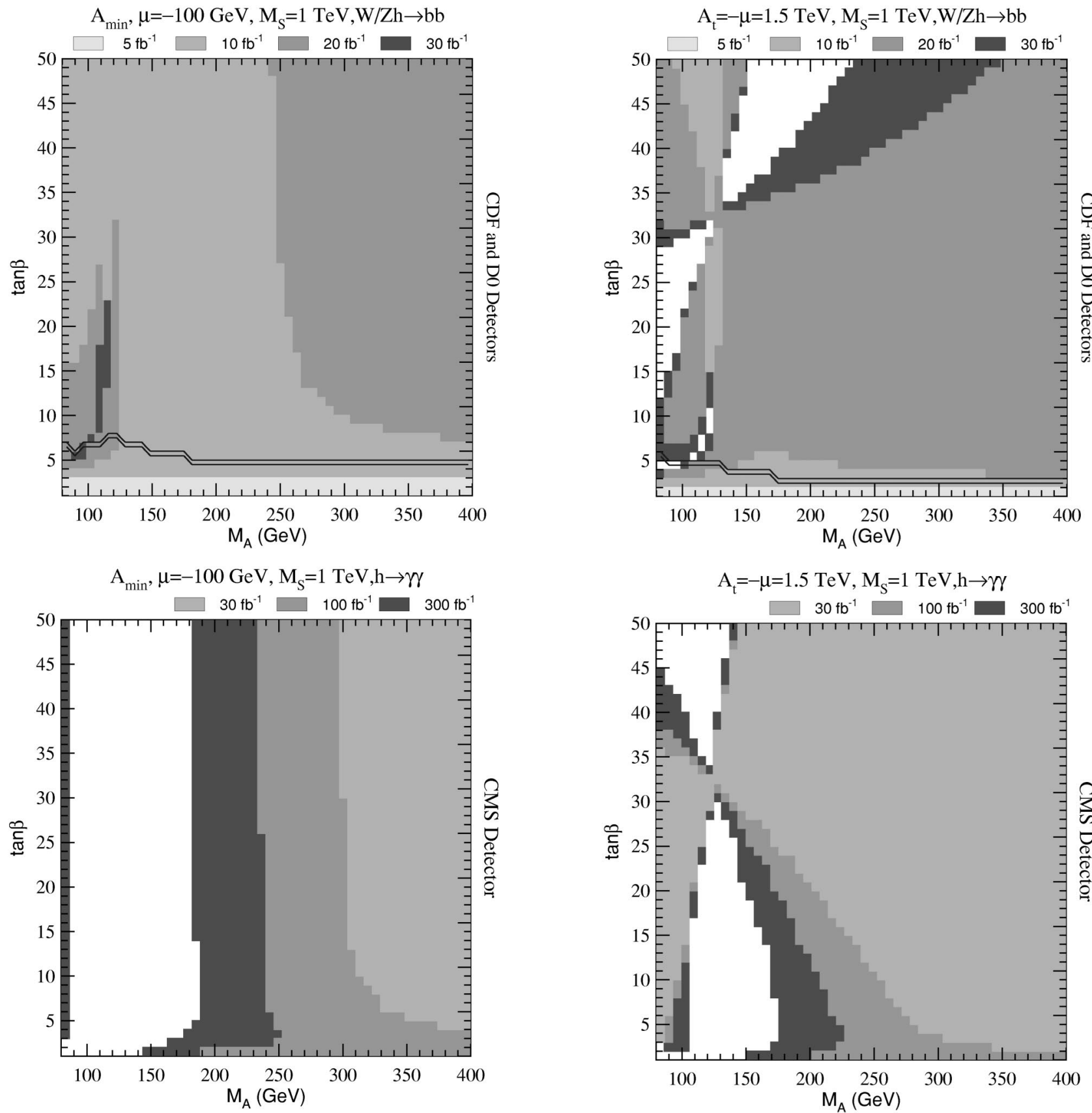

FIG. 2. Discovery reach of the LEP, Tevatron and LHC experiments in the minimal mixing case, as defined in the text.

\section{B. $\phi_{W} b \bar{b}$ suppression for large $M_{S}$ by corrections to the Higgs boson mass matrix}

Figure 3 was generated using the choice of MSSM parameters $A_{t}=-\mu=1.5 \mathrm{TeV}$ and $M_{S}=1 \mathrm{TeV}$, which yields a suppression of the Higgs boson coupling to $b \bar{b}$ (and $\tau^{+} \tau^{-}$) in a significant portion of the $m_{A}$-tan $\beta$ plane for the same Higgs boson that couples strongly to $W$ and $Z$ bosons $\left(\phi_{W}\right)$. An approximate analytic formula that shows the necessary relations between parameters was presented in Ref. [24]. In Fig. 3(a), the vanishing of $\sin \alpha$ is seen when $h \equiv \phi_{W}$ (region which remains uncovered by the Tevatron, in the upper part

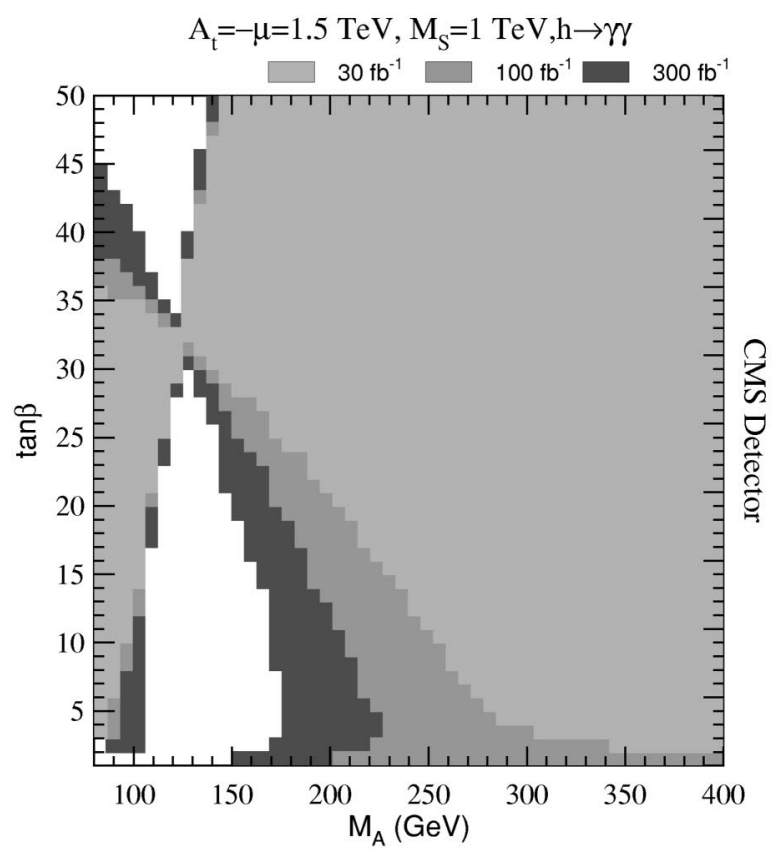

FIG. 3. Same as Fig. 2 but for $A_{t}=-\mu=1.5 \mathrm{TeV}, M_{S}$ $=1 \mathrm{TeV}$.

of the figure), whereas the vanishing of $\cos \alpha$ occurs when $H \equiv \phi_{W}$ (region which remains uncovered by the Tevatron, in the lower part of the figure). As alluded to earlier, the vanishing of the $\phi_{W} b \bar{b}$ and $\phi_{W} \tau^{+} \tau^{-}$couplings greatly enhances $\operatorname{BR}\left(\phi_{W} \rightarrow \gamma \gamma\right)$, and CMS has little difficulty in covering these regions in the low luminosity run, as shown in Fig. 3(b).

After combining both discovery reaches, a small region, for which $\sin ^{2}(\beta-\alpha) \simeq \cos ^{2}(\beta-\alpha)$ persists, however, uncovered by both colliders. For clarity, we wish to emphasize again that LHC will have other means of testing the region of parameters which remain uncovered in our analysis by 
means of the production of other Higgs bosons [16]. However, in this region of parameters, none of these Higgs bosons will have SM-like couplings, and hence will not directly test the mechanism of electroweak symmetry breaking.

\section{Suppression of the $\phi_{W} b \bar{b}$ including the direct corrections to the bottom quark Yukawa coupling}

So far, our analysis has neglected corrections to the fermion Yukawa couplings from loop corrections. To illustrate the potential importance of these effects, we must specify other parameters of the MSSM which, in the next-to-leadinglogarithmic approximation, do not affect the $C P$-even Higgs boson masses and mixing angles in a significant way (apart from the effects arising from the redefinition of the $b$-quark Yukawa coupling, which we will discuss below). Care must be taken in choosing these parameters, since an additional enhancement of the $b$-quark Yukawa coupling at large values of $\tan \beta$ can cause the theory to become non-perturbative. Therefore, we consider an example similar to the one presented in Fig. 3, taking $A_{t}=-\mu=1 \mathrm{TeV}$. This still leads to a suppression of the $\phi_{W} b \bar{b}$ coupling via the quantum corrections to the $C P$-even Higgs boson mass matrix, but in a smaller region of the $M_{A}$-tan $\beta$ plane. For this same choice of parameters, we then consider the effects of $\Delta\left(m_{b}\right)$ when the gluino mass parameter $M_{\tilde{g}}$ takes the values $\pm .5 \mathrm{TeV}$. From the expression for $\Delta\left(m_{b}\right)$ (see Ref. [24], for example), we observe that the top-quark-Yukawa-coupling-induced contribution is negative for $A_{t} \mu<0$, while a positive (negative) gluino mass will decrease (increase) $\Delta\left(m_{b}\right)$. The case of an overall positive correction (negative gluino mass) is illustrated in Fig. 4. The effect of the correction is minimal, since $\Delta\left(m_{b}\right)$ is never larger than about 0.3 at large $\tan \beta$. For the case of a negative correction, however, as seen in Fig. 5, the suppression of the $\phi_{W} b \bar{b}$ coupling for $m_{A} \geq 130 \mathrm{GeV}$ is shifted to lower values of $\tan \beta$. Note that the suppression is now occurring because $\tan \alpha \simeq \Delta\left(m_{b}\right) / \tan \beta$, and not because the tree-level coupling, $\sin \alpha \cos \alpha / \cos \beta$, vanishes.

According to Eq. (3.7), in the regions of parameter space where the tree-level $\phi_{W} b \bar{b}$ vanishes, the full $\phi_{W} b \bar{b}$ coupling can still be substantial, but the $\phi_{W} \tau^{+} \tau^{-}$coupling is suppressed. When the $\phi_{W} b \bar{b}$ coupling suppression occurs, the $\phi_{W} \tau^{+} \tau^{-}$coupling will typically not vanish, as demonstrated in Eq. (3.9). There are several phenomenological consequences of the mismatch in the behavior of the $\phi_{W} b \bar{b}$ and $\phi_{W} \tau^{+} \tau^{-}$couplings. In the previous examples, the simultaneous vanishing of the $\phi_{W} b \bar{b}$ and the $\phi_{W} \tau^{+} \tau^{-}$couplings led to an enhancement of $\operatorname{BR}\left(\phi_{W} \rightarrow \gamma \gamma\right)$. However, the enhancement of $\operatorname{BR}\left(\phi_{W} \rightarrow \gamma \gamma\right)$ in the region where $\phi_{W} b \bar{b}$ is suppressed will generally not be as large as naively expected, since, as can be inferred from Eq. (3.9), $\Gamma\left(\phi_{W} \rightarrow \tau^{+} \tau^{-}\right)$can still be substantial. Still, the enhancement of $\mathrm{BR}\left(\phi_{W} \rightarrow \gamma \gamma\right)$ will be sufficient, so that complementarity of the Tevatron and the LHC experiments in the search for the Higgs $\phi_{W}$ boson remains clear. Given the fact that the decay $\phi_{W}$ $\rightarrow \tau^{+} \tau^{-}$may be the dominant one in this region of param-
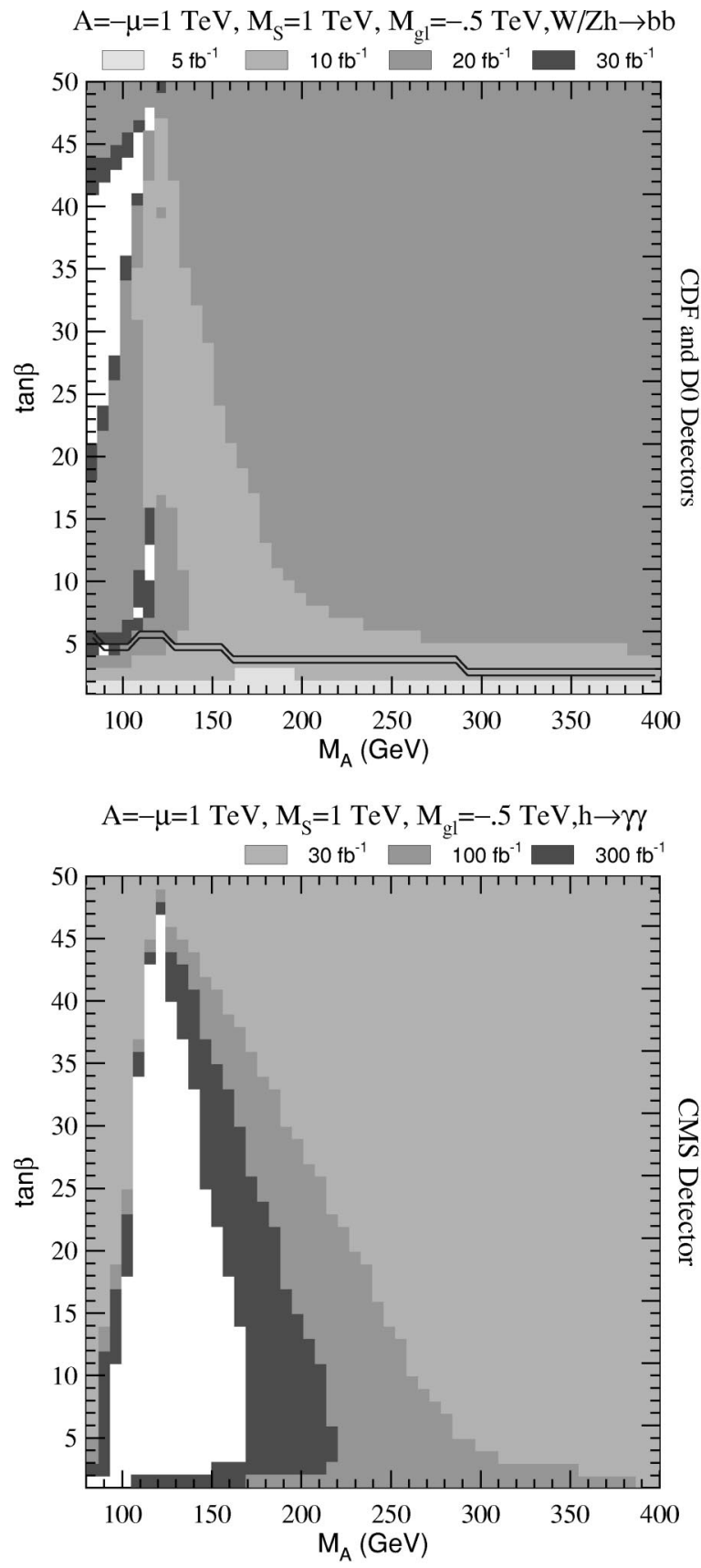

FIG. 4. Same as Fig. 2, but for $A_{t}=-\mu=1 \mathrm{TeV}$, and including the effects of the bottom mass corrections, $\Delta\left(m_{b}\right)$, calculated using $M_{\tilde{g}}=-.5 \mathrm{TeV}$.

eters, it is important to consider $\phi_{W} \rightarrow \tau^{+} \tau^{-}$signatures at the Tevatron and LHC. Preliminary results in this direction have been presented in Ref. [14].

An illustration of the possible variation of the $\phi_{W} \rightarrow b \bar{b}$ and $\phi_{W} \rightarrow \tau^{+} \tau^{-}$decay modes in parameter space is presented in Fig. 6, which shows the function $R$ of Eq. (3.3) for the $b \bar{b}$ final state $\left(R_{b}\right)$ (a) and the $\tau^{+} \tau^{-}$final state $\left(R_{\tau}\right)$ (b) for the same parameters as in Fig. 5. Note the large region where $R_{b}$ is greater than the SM value, whereas $R_{\tau}$ is below it. Of course, there is another region along contours of $\tan \alpha \simeq \Delta\left(m_{b}\right) / \tan \beta$ at very large $\tan \beta$ where $\phi_{W} \rightarrow \tau^{+} \tau^{-}$is the dominant decay mode. In terms of area covered in the 

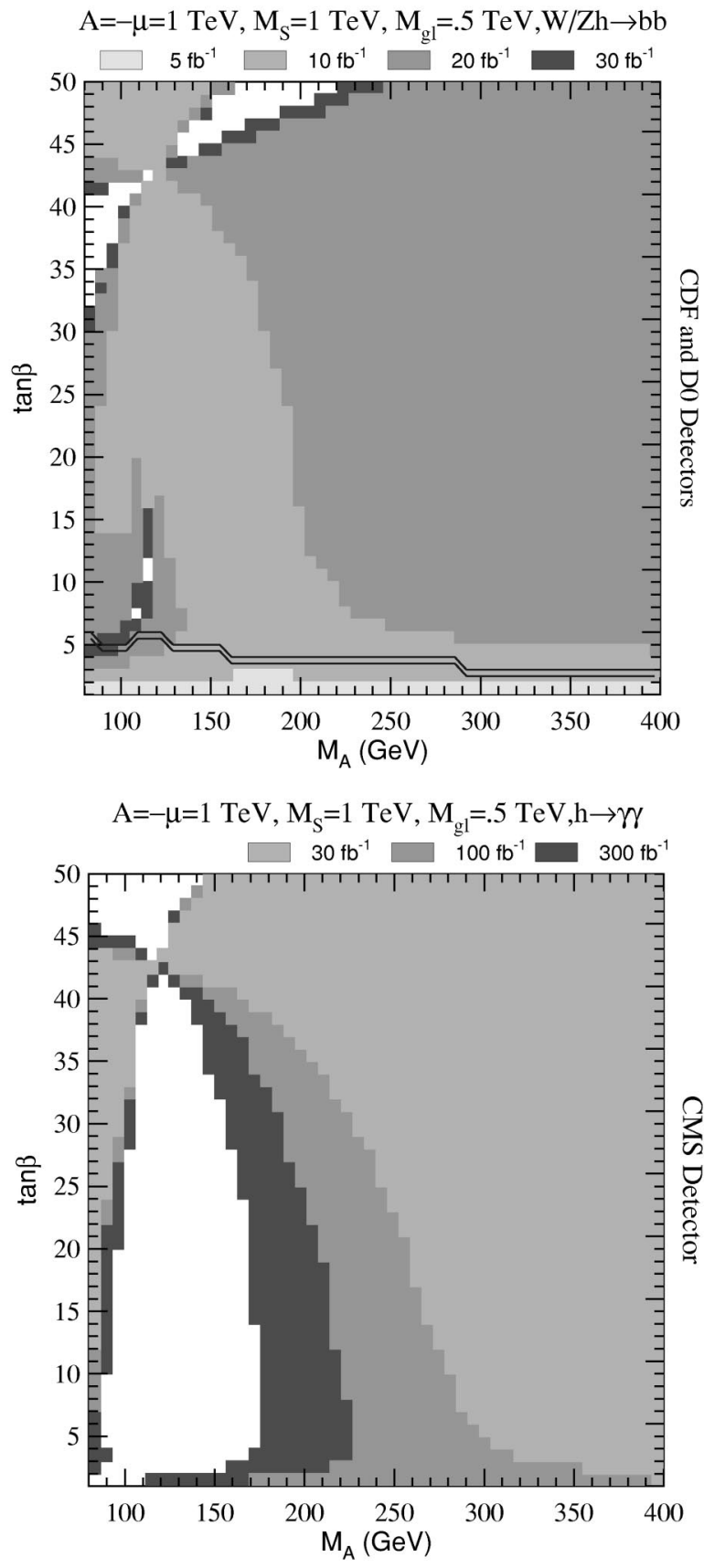

FIG. 5. Same as Fig. 4, but for $M_{\tilde{g}}=.5 \mathrm{TeV}$.

$M_{A}$-tan $\beta$ plane, the former is more significant than the latter. It is also worth noting that both $R$ factors, Eq. (3.2), approach their SM values in the limit of large $M_{A}$. Indeed, when $M_{A}$ is large and $M_{S}$ is large, the effect of the radiative corrections vanishes. When $M_{A}$ is fixed and $M_{S}$ is arbitrarily large, however, the radiative corrections will in general remain relevant [24], a reflection of the lack of supersymmetry in the low energy effective theory.

It is important to emphasize that the nice complementarity between the $\phi_{W} \rightarrow b \bar{b}$ and $\phi_{W} \rightarrow \gamma \gamma$ decay channels holds due to the fact that the former is, in general, the dominant Higgs boson decay channel. This complementarity does not extend to the $\phi_{W} \rightarrow \tau^{+} \tau^{-}$channel. Indeed, in the above ex-
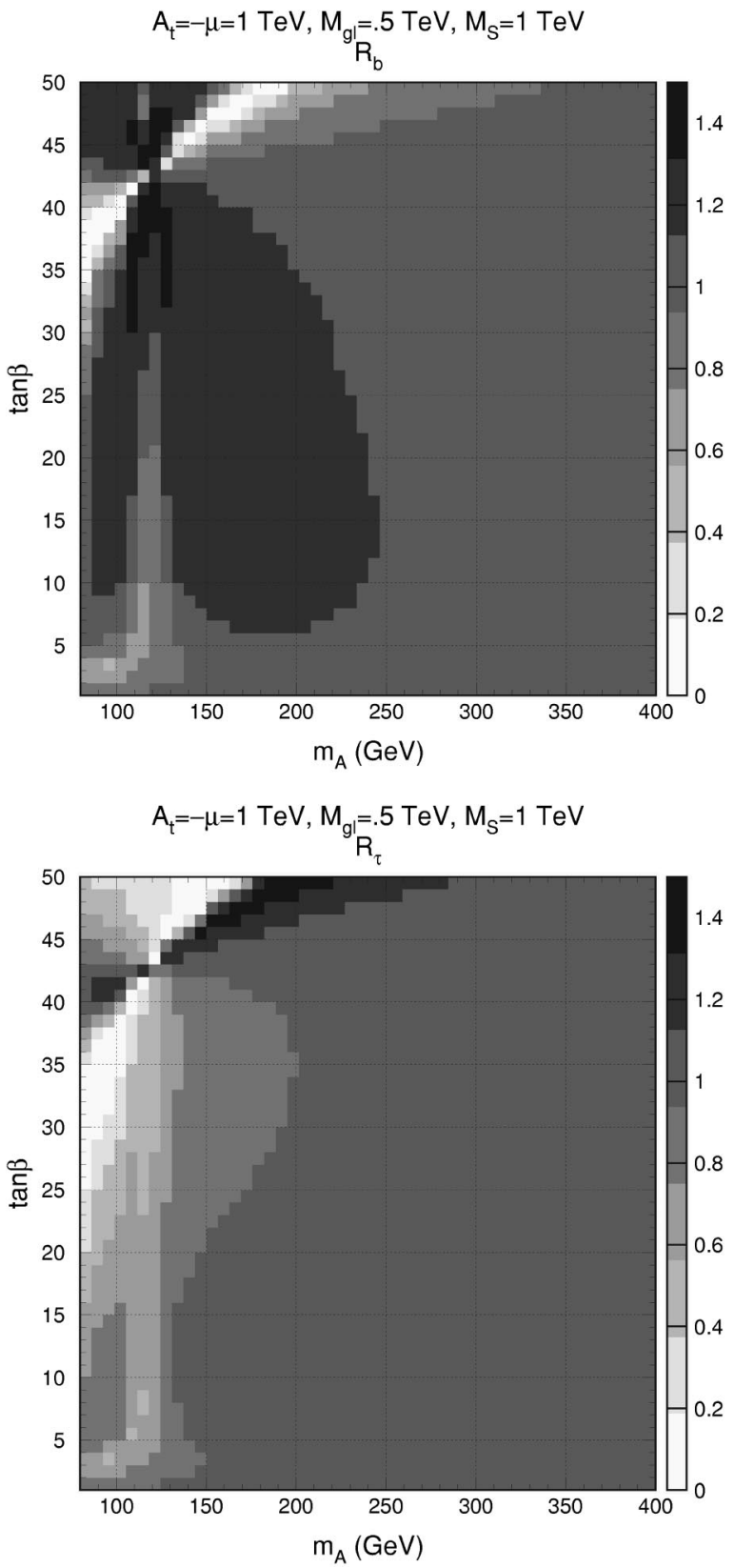

FIG. 6. Comparison of the sensitivity of the $\phi_{W} \rightarrow b \bar{b}$ and $\phi_{W}$ $\rightarrow \tau^{+} \tau^{-}$channels, measured by the function $R$ defined in Eq. (3.2), applying it for the $b \bar{b}$ and also the $\tau^{+} \tau^{-}$final states, $R_{b}$ and $R_{\tau}$, respectively, when the Higgs $\phi_{W}$ is produced from a $V V \phi_{W}$ vertex, with $V=W$ or $Z$.

ample, we see the region of parameters for which $\phi_{W}$ $\rightarrow \tau^{+} \tau^{-}$is suppressed, and as a result of a slight enhancement of $\phi_{W} \rightarrow b \bar{b}$ decay rate in the same region of parameters, the LHC reach in the $\phi_{W} \rightarrow \gamma \gamma$ channel is also suppressed.

\section{Effects of the bottom squark sector}

In the previous examples, we showed how relatively large values of $A_{t} \simeq-\mu$ led to the suppression of the $\phi_{W} b \bar{b}$ coupling in the large $\tan \beta$ regime. We demonstrate that this can 

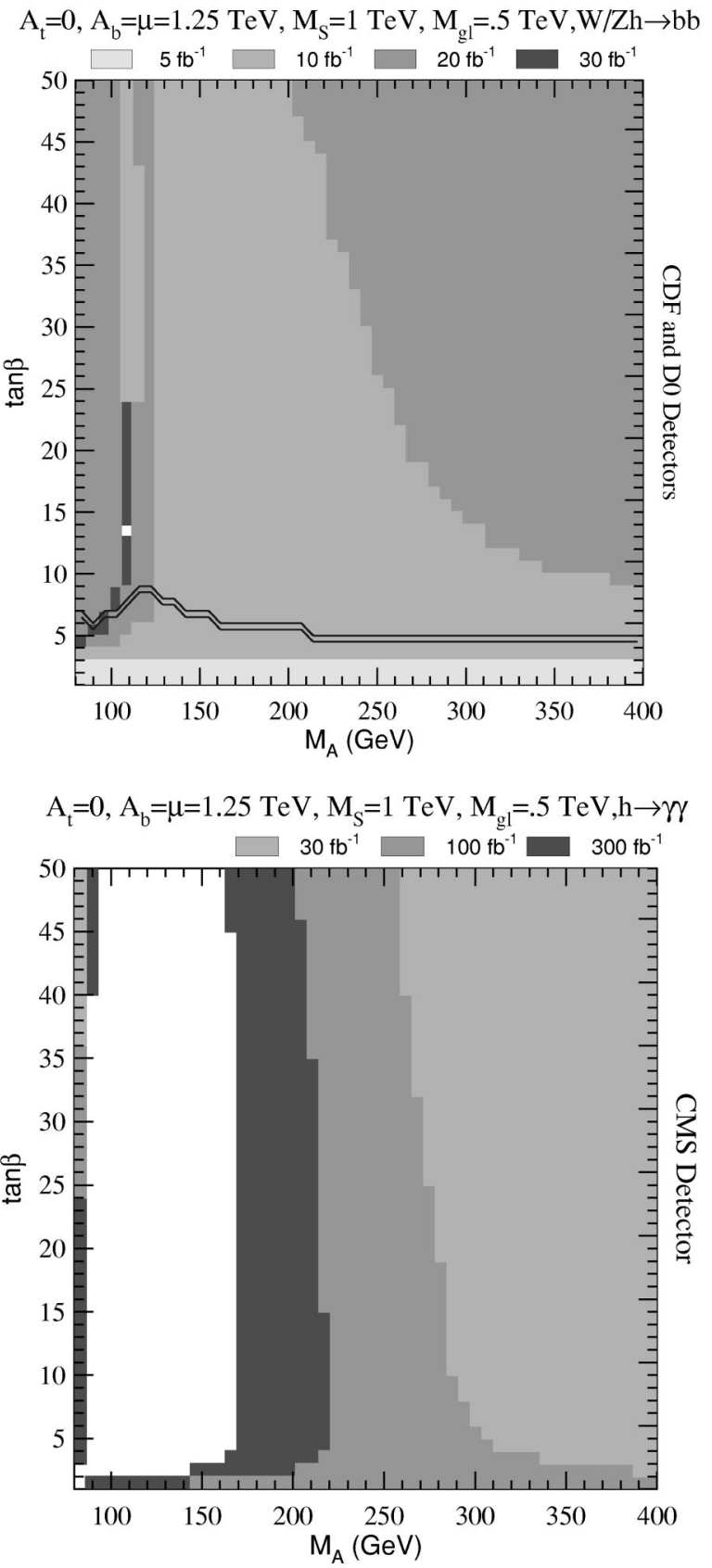

FIG. 7. Same as Fig. 2, but considering a possible suppression of $\operatorname{BR}\left(\phi_{W} \rightarrow b \bar{b}\right)$ for large $A_{b}=\mu$ and $\Delta\left(m_{b}\right)>0$.

also occur when $A_{b} \simeq \mu$ in Figs. 7 and 8 . The effect of $A_{b}$, however, is in general much weaker than the $A_{t}$ one, and becomes only relevant for large values of the bottom quark Yukawa coupling - that is, for values of $\tan \beta \simeq m_{t} / m_{b}$. To show this, in these figures, we choose moderate values of $A_{b}$ and $\mu$, taking $A_{t}=0$. The vanishing of $A_{t}$ leads, in general, to masses that are of the order of the ones obtained in the minimal mixing case, although they can be further reduced, due to the $\mu$-induced terms discussed above, for large values of $\tan \beta$. Also, the top quark Yukawa contribution to $\Delta\left(m_{b}\right)$ vanishes for $A_{t}=0$. In Fig. 7, we have chosen $A_{b}=\mu$ $=1.25 \mathrm{TeV}$ and $M_{\tilde{g}}=.5 \mathrm{TeV}$, leading to $\Delta\left(m_{b}\right)>0$. No relevant modification in the reach is found compared to the
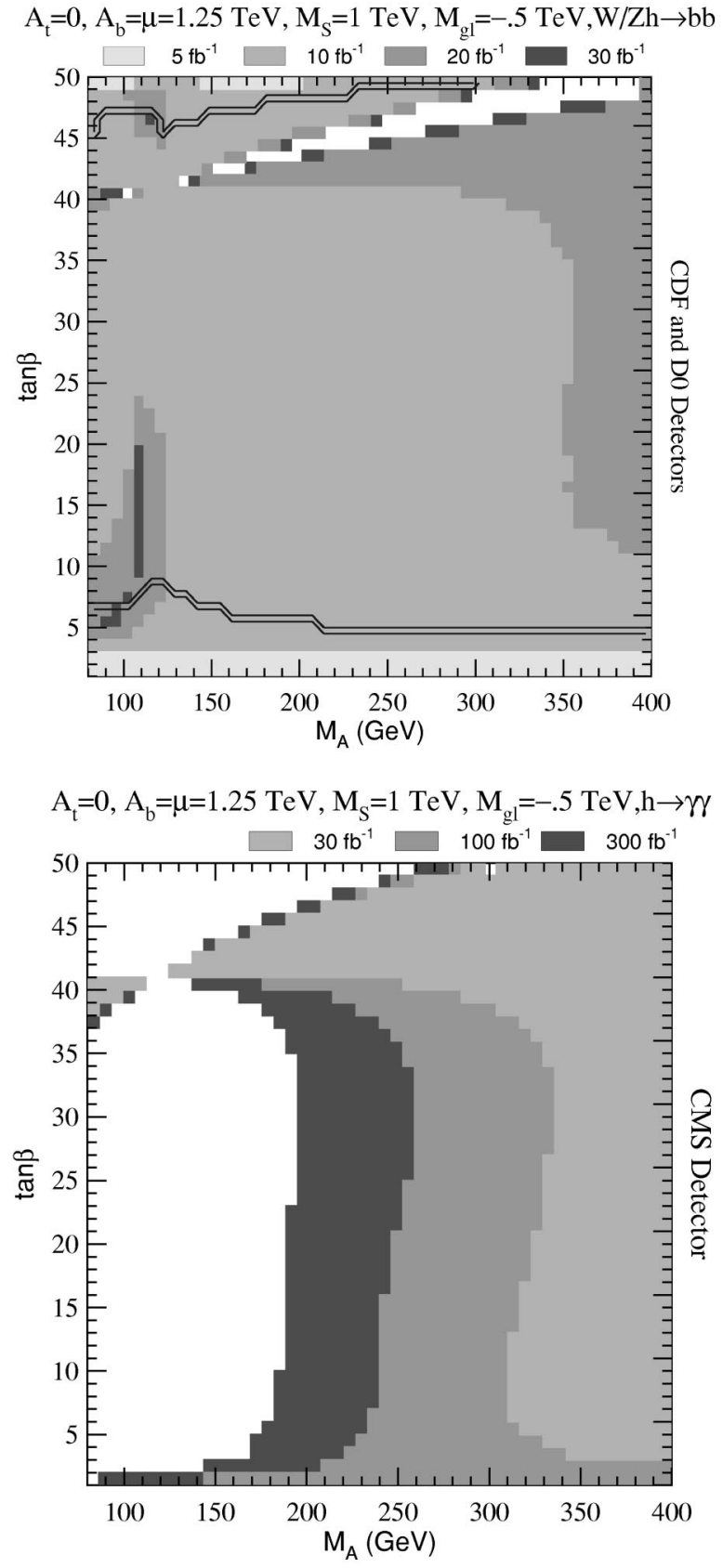

FIG. 8. Same as Fig. 7 but for $\Delta\left(m_{b}\right)<0$.

minimal mixing case (Fig. 2). Indeed, for the parameters chosen, the suppression of the $\phi_{W} b \bar{b}$ coupling takes place at values of $\tan \beta$ larger than the ones considered in this analysis. To observe the suppression for positive corrections, we would need to choose larger values of $A_{b}$ and $\mu$.

For $M_{\tilde{g}}=-.5 \mathrm{TeV}$, which yields $\Delta\left(m_{b}\right)<0$, we observe the suppression of the $\phi_{W} b \bar{b}$ coupling in Fig. 8, in the region of parameter space in which $\tan \alpha \simeq \Delta\left(m_{b}\right) / \tan \beta$. This leads to the existence of regions of parameter space where $\phi_{W}$ is unaccessible to the LEP and the Tevatron colliders, but it will be easily observed at LHC, due to an increase of the $\phi_{W} \rightarrow \gamma \gamma$ rate in the same regions of parameter space. Since we have also included the bottom quark Yukawa coupling corrections to the calculation of the mass spectrum, we ob- 
serve the reappearance of the LEP exclusion at large $\tan \beta$, due to a reduction of the Higgs boson mass. If we compare the LHC reach with that obtained in Fig. 2(b), we see that for large values of $\tan \beta$ the LHC reach is quite different from the one obtained in the minimal mixing case. Indeed, while in the regions where the $\phi_{W} b \bar{b}$ coupling is suppressed, the LHC reach is enhanced, as expected, the region of parameter space for which the Higgs $\phi_{W}$ boson becomes unaccessible for LHC extends to larger values of $m_{A}$. This is mainly due to the reduction of the $\phi_{W}$ boson mass mentioned above, which enhances the reach of LEP and the Tevatron collider, but makes the $\phi_{W}$ searches at LHC more difficult.

Although the above provides only an extreme example for which only the $A_{b}$-induced effects were considered, it shows in a clear way the relative importance of the effects on the Higgs boson mass matrix elements induced by the presence of non-trivial mixing in the bottom and top squark sectors. In the following sections, we shall present examples in which $A_{t} \simeq A_{b} \neq 0$.

\section{E. Light top squark and large mixing}

In the minimal mixing model investigated in Sec. IV A, the SUSY scale $M_{S}$ was set to $1 \mathrm{TeV}$, but the mixing term $\widetilde{A}_{t}$ was zero. In the present case, we consider equal values for the left- and right-handed soft-supersymmetry-breaking squark masses of the third generation, and adjust their common value to yield a lightest top squark mass of $200 \mathrm{GeV}$. In this section, as in the following ones, we shall ignore the effects of $\Delta\left(m_{b}\right)$, or, equivalently, we shall assume that the gluino mass parameter value takes on a value so that the total bottom quark mass correction is small. We fix $\mu=$ $-.3 \mathrm{TeV}$ and $A_{t}=1 \mathrm{TeV}$, so that top squark mixing is large. ${ }^{7}$ This is meant to demonstrate the possibility of large corrections to the $\phi_{W} g g$ and $\phi_{W} \gamma \gamma$ couplings from light sparticles. This is motivated by the form of the $\{h, H\} \tilde{t} \tilde{t}$ coupling (written here in the interaction basis):

$$
\begin{gathered}
{\left[\frac{2 m_{t}^{2}}{v \sin \beta}\{\cos \alpha, \sin \alpha\}-\frac{2 M_{Z}^{2}}{v}\{\sin (\alpha+\beta),-\cos (\alpha+\beta)\}\right.} \\
\left.\times\left(1 / 2-2 / 3 \sin ^{2} \theta_{W}\right)\right](L L) \\
{\left[\frac{2 m_{t}^{2}}{v \sin \beta}\{\cos \alpha, \sin \alpha\}-\frac{2 M_{Z}^{2}}{v}\{\sin (\alpha+\beta),\right.} \\
\left.\quad-\cos (\alpha+\beta)\} 2 / 3 \sin ^{2} \theta_{W}\right] \quad(R R) \\
\frac{m_{t}}{v \sin \beta}\left[A_{t}\{\cos \alpha, \sin \alpha\}+\mu\{\sin \alpha,-\cos \alpha\}\right] \quad(L R)
\end{gathered}
$$

\footnotetext{
${ }^{7}$ As has been observed in Ref. [29], precision electroweak measurements tend to disfavor the presence of light third generation squarks with large mixing angles. Most of the parameters considered in this subsection, as in the following two, are only marginally consistent with the precision electroweak data.
}

where we have denoted the components in parentheses. For large $L R$ mixing, the terms proportional to $A_{t}$ and $\mu$ can dominate the top-squark-Higgs-boson couplings. For a Higgs boson with SM-like couplings to the gauge bosons, in the moderate and large $\tan \beta$ regime, $A_{t}$ is the relevant mixing parameter determining the strength of these couplings. For large values of $m_{A}$ and arbitrary values of $\tan \beta$, this coupling is proportional to $\widetilde{A}_{t}$, which is approximately equal to $A_{t}$ in the large $\tan \beta$ regime.

For such a choice of the top squark sector parameters, the Tevatron can discover a Higgs $\phi_{W}$ boson in most of the $M_{A}-\tan \beta$ plane, with about $20 \mathrm{fb}^{-1}$. One observes from Fig. 9(a) that although the top squarks are lighter in this case, the reach at the Tevatron is somewhat suppressed with respect to the minimal mixing case, and $30 \mathrm{fb}^{-1}$ are necessary to cover the whole parameter space, with the exception of the region of parameters for which $\sin ^{2}(\beta-\alpha) \simeq \cos ^{2}(\beta-\alpha)$. The origin of the relative suppression in the discovery reach is the upper bound on the lightest $C P$-even Higgs boson mass, which increases substantially when $\widetilde{A}_{t} \neq 0$. For instance, while for the minimal mixing case this upper bound is below $115 \mathrm{GeV}$, values close to $120 \mathrm{GeV}$ are obtained in the case under analysis.

As noted earlier, light top and bottom squarks can have a large effect on the one-loop suppressed partial widths $\Gamma\left(\phi_{W} \rightarrow g g\right)$ and $\Gamma\left(\phi_{W} \rightarrow \gamma \gamma\right)$. When the top squark mixing mass parameters (in particular $A_{t}$ ) become large, the width $\Gamma\left(\phi_{W} \longrightarrow g g\right)$ can be greatly decreased since the light top squark loop contribution can partially or totally cancel the top quark loop induced one. In Fig. 9(b), it is clear that $R^{\prime}$, Eq. (3.10), is strongly suppressed, and this is reflected in the fact that the low luminosity run of the LHC cannot observe the Higgs boson that couples strongly to the $W$ and $Z$ boson in the gluon fusion channel. The high luminosity run relies to a lesser extent on $\Gamma\left(\phi_{W} \rightarrow g g\right)$, and the reach is dominated by the $t \bar{t} \phi_{W} / W \phi_{W}$ channels (see also [15]). In this Higgs boson mass range, the LHC sensitivity in these channels depends only weakly on the Higgs boson mass and for high luminosity the LHC discovery reach becomes similar to the one in the minimal mixing case.

The complementarity of the Tevatron and LHC colliders in this case is similar to the case of minimal mixing, although somewhat larger luminosities in both colliders are needed in order to obtain full coverage of the MSSM parameter space.

\section{F. $\phi_{W} b \bar{b}$ coupling suppression for light top squarks}

Since we have identified two interesting effects, namely the suppression of the $\phi_{W} b \bar{b}$ coupling due to Higgs boson mixing and the suppression of one-loop couplings from large top squark mixing, one may wonder if both can occur simultaneously. The conditions for the cancellation of $\mathcal{M}_{12}^{2}$ in the case of large top squark mixing effects, and hence for $\phi_{W} b \bar{b}$ suppression, can be determined analogously to the large $M_{S}$ case, but since the top squark mixing effects become larger, one should work with the full effective potential computation [7]. We display an example in which both the $\phi_{W} b \bar{b}$ cou- 

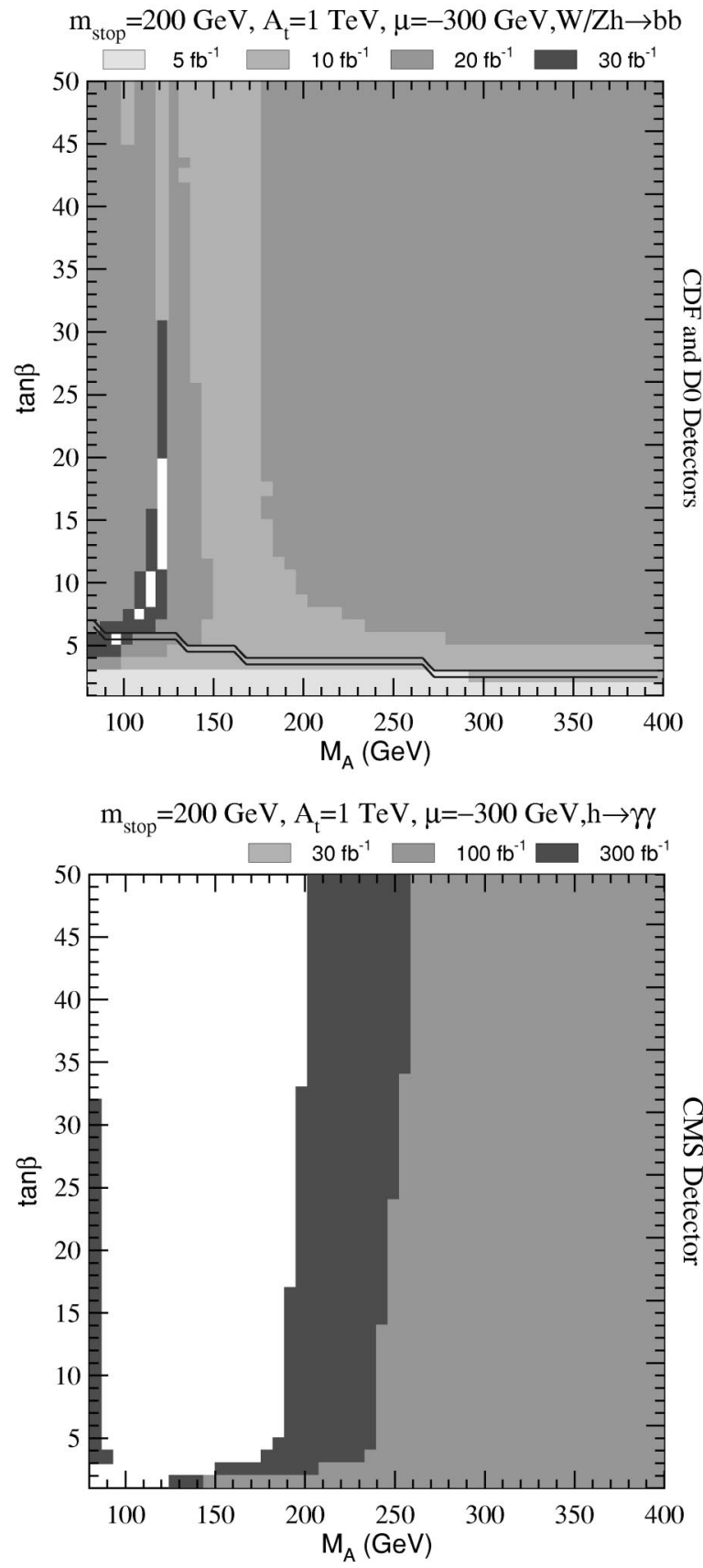

FIG. 9. Same as Fig. 2, but for a region of parameters such that $\Gamma\left(\phi_{W} \rightarrow g g\right)$ is suppressed with respect to the SM value, $M_{\tilde{t}_{1}}$ $=200 \mathrm{GeV}, A_{t}=1 \mathrm{TeV}, \mu=-.3 \mathrm{TeV}$.

pling and the one loop couplings of $\phi_{W}$ to gluons are suppressed in Fig. 10. In this example $\mu=1 \mathrm{TeV}, A_{t}$ $=.65 \mathrm{TeV}$, and the lightest top squark mass fixed at 200 $\mathrm{GeV}$ as before. Since the top squark mixing effects we are considering are larger than in the previous cases, the cancellation of the $\phi_{W} b \bar{b}$ coupling occurs for larger values of the $C P$-odd Higgs boson mass. Indeed, Fig. 10(a) reveals that the region of $\phi_{W} b \bar{b}$ suppression is shifted substantially.

Most interesting is the fact that LEP could discover a Higgs boson with strong couplings to $W$ and $Z$ bosons at large $\tan \beta$. As noticed before, this is just a reflection of the
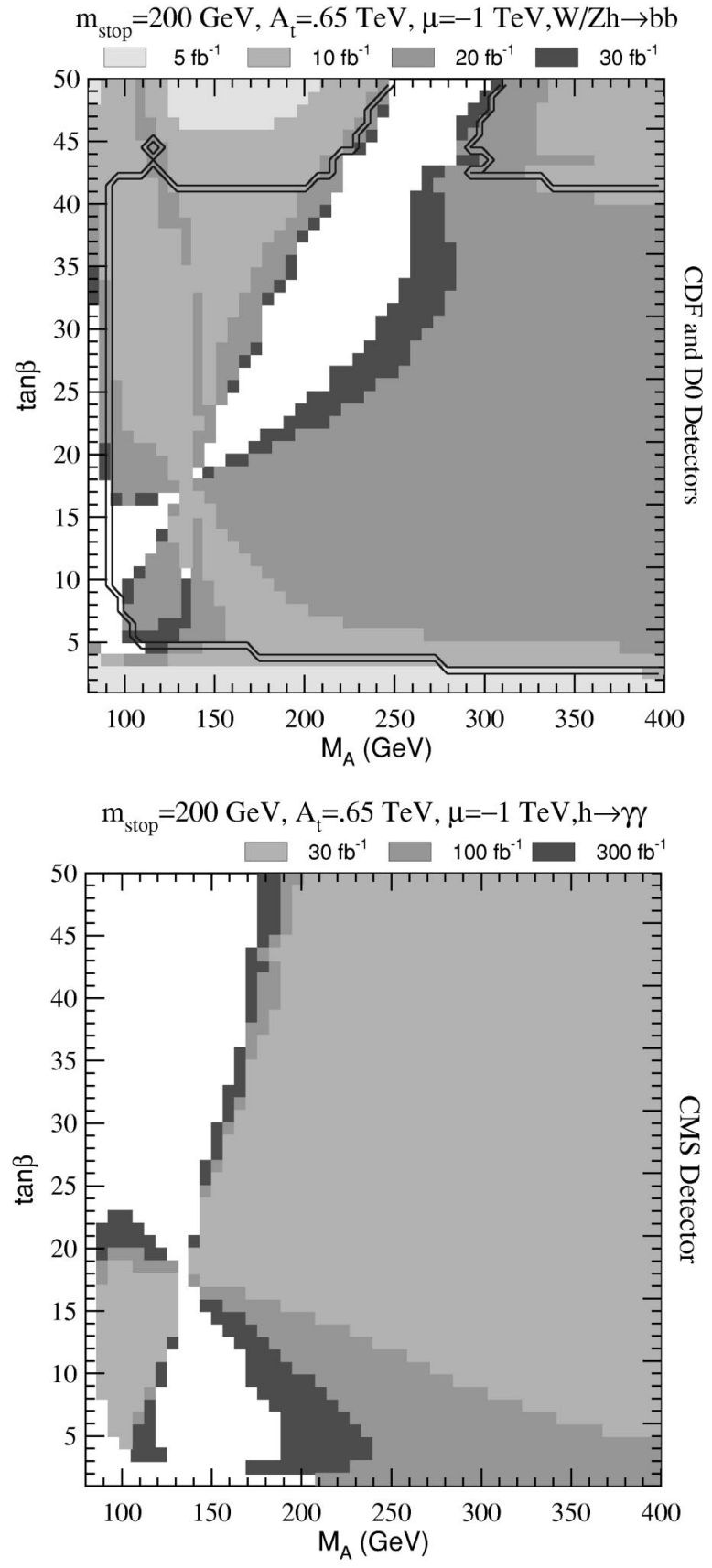

FIG. 10. Same as Fig. 9, but considering top squark mixing mass parameters which induce a suppression of the $\phi_{W} b \bar{b}$ coupling, $M_{\tilde{t}_{1}}=200 \mathrm{GeV}, A_{t}=.65 \mathrm{TeV}, \mu=-1 \mathrm{TeV}$.

suppression of the Higgs boson mass induced by the $\mu$ parameter at large values of $\tan \beta$ [see the expression for $\mathcal{M}_{22}^{2}$ in Ref. [24]). Of course, in the regions of $\phi_{W} b \bar{b}$ suppression, neither LEP nor the Tevatron has any reach (we are not considering a possible enhancement of the tau lepton coupling, which, as explained before, can take place if $\Delta\left(m_{b}\right)$ $\left.\neq \Delta\left(m_{\tau}\right)\right]$.

Because of the suppression of the $\phi_{W} b \bar{b}$ coupling, the LHC, Fig. 10(b), has significant reach in the regions where LEP and the Tevatron cannot detect $\phi_{W}$. Observe that the 
reach at low $\tan \beta$ and large $m_{A} \geqslant 350 \mathrm{GeV}$ is slightly less than for the minimal mixing model, because of a small increase of the $\phi_{W} b \bar{b}$ coupling. However, for the same values of $\tan \beta$ and $M_{A}$, the lightest $C P$-even Higgs boson mass increases, and therefore the increase of the $\operatorname{BR}(h \rightarrow b \bar{b})$ is not reflected in an increase of the Tevatron reach.

More important, for large values of $\tan \beta$ there are regions of the parameter space for which the Higgs boson becomes accessible to the three colliders with relatively small luminosity. This would provide a perfect situation: A Higgs boson with SM-like couplings to the gauge bosons will be discovered at LEP by the end of the year 2000, and its properties will be further tested at the Tevatron and LHC colliders.

\section{G. $\phi_{W} b \bar{b}$ coupling enhancement and light third generation squarks}

A large value of $\widetilde{A}_{b} \equiv A_{b}-\mu \tan \beta$ can have significant consequences when the bottom squark is also light. In Fig. 11(b), one observes a general suppression of $R^{\prime}$ and $R^{\prime \prime}$ throughout most of the $M_{A}$-tan $\beta$ plane. For this example, we have set $A_{t}=A_{b}=-0.5 \mathrm{TeV}, \mu=-1 \mathrm{TeV}$, and all third generation squark parameters equal and tuned to yield a bottom squark mass of $200 \mathrm{GeV}$. For small values of the ratio of Higgs vacuum expectation values $(\tan \beta<2)$ this choice of parameters may lead to top squarks with masses below the present experimental bound or unphysical ones. In these cases, we have increased the squark masses by setting a lower bound on the lightest bottom squark of about 300 $\mathrm{GeV}$. Although this implies a slight discontinuity of the parameters chosen to make the figures, this does not affect the physical results, since for such light stops and sbottoms the $\tan \beta<2$ regime is already ruled out by LEP 2 data.

Since $\widetilde{A}_{t}$ and $\widetilde{A}_{b}$ are large, and the bottom and top squarks are light, $\Gamma\left(\phi_{W} \rightarrow g g\right)$ will decrease and $\Gamma\left(\phi_{W} \rightarrow \gamma \gamma\right)$ will increase. However, the $\operatorname{BR}\left(\phi_{W} \rightarrow \gamma \gamma\right)$ does not increase due to the fact that, for this choice of parameters, the bottom quark coupling to the Higgs $\phi_{W}$ boson is enhanced with respect to the standard model expectation, inducing a decrease of the $\operatorname{BR}\left(\phi_{W} \rightarrow \gamma \gamma\right)$. Had we chosen the opposite sign of $\mu$, an increase of the $\operatorname{BR}\left(\phi_{W} \rightarrow \gamma \gamma\right)$ with respect to the SM value would have been observed, as in the previous section.

Because of the enhancement of $\operatorname{BR}\left(\phi_{W} \rightarrow b \bar{b}\right)$ and given that the Higgs $\phi_{W}$ boson mass is below $112 \mathrm{GeV}$ (it becomes smaller at small and large values of $\tan \beta$ ), it is particularly easy to probe this region of the MSSM parameters. For instance, LEP can probe a significant portion of the $M_{A}$-tan $\beta$ plane (LEP will be sensitive to the region connected by the narrow strip around $M_{A} \simeq 135 \mathrm{GeV}$ ) as seen in Fig. 11(a), and the Tevatron would only need a substantial luminosity upgrade to cover the difficult region near $M_{A} \simeq 120 \mathrm{GeV}$.

\section{CONCLUSIONS}

If the breakdown of electroweak symmetry is induced by the vacuum expectation value of fundamental scalar fields,
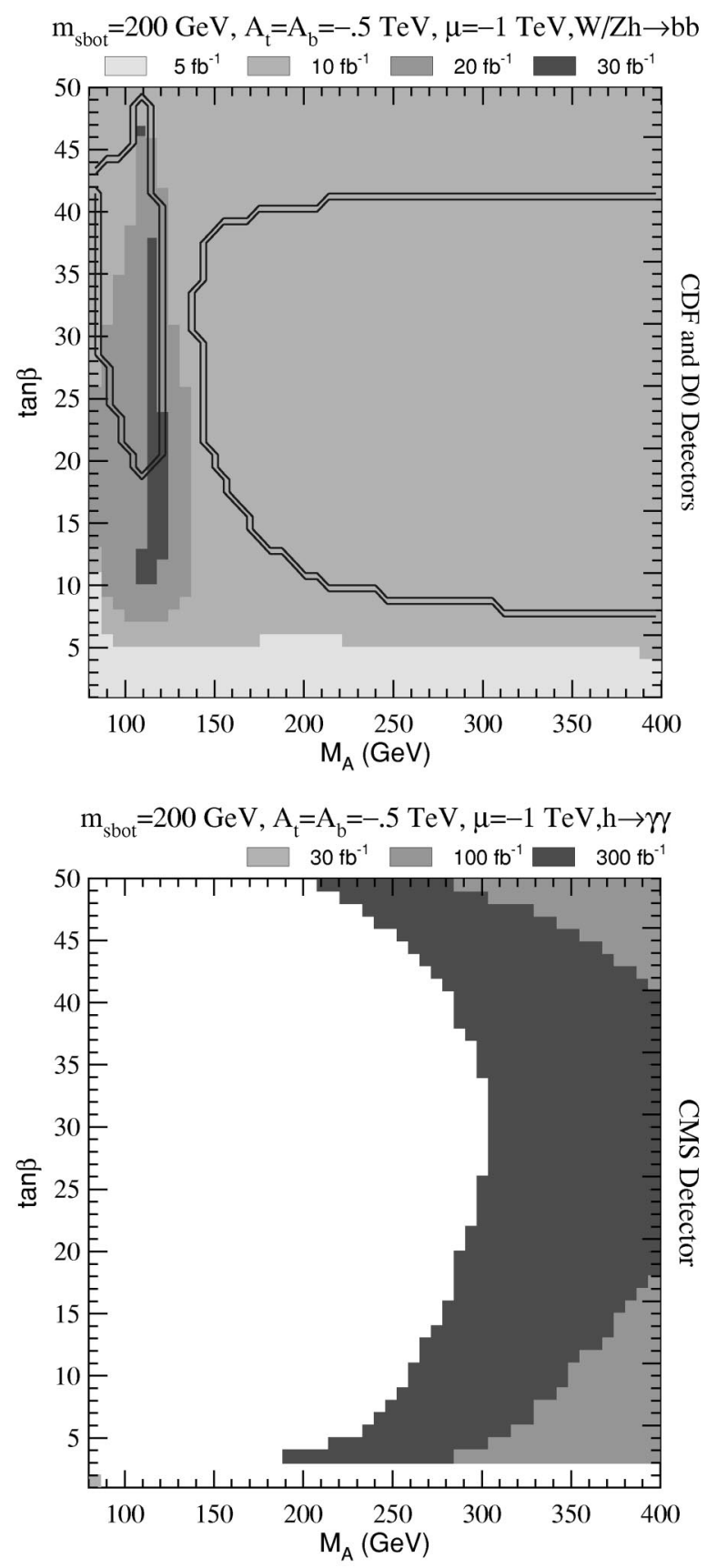

FIG. 11. Same as Fig. 2, but for light top and bottom squarks, and large mixing mass parameters, $M_{\tilde{b}_{1}}=200 \mathrm{GeV}, A_{t}=A_{b}=$ $-.5 \mathrm{TeV}, \mu=-1 \mathrm{TeV}$.

the presence of at least one physical neutral Higgs boson, with couplings to the weak gauge bosons which are not much smaller than the standard model one, is to be expected. In theories with more than one Higgs doublet, the real part of the neutral Higgs combination which acquires vacuum expectation value is not necessarily associated with a physical mass eigenstate. In the MSSM, however, there are large regions of the parameter space where one of the $C P$-even Higgs bosons couples in a standard model way to the $W$ and $Z$ bosons and up quarks and, hence, can be identified as the dominant source of electroweak symmetry breaking. 
In this work, we have denoted the lightest $C P$-even Higgs boson which has the largest coupling to the weak gauge bosons as $\phi_{W}$, and we have concentrated on searches by means of only the standard model Higgs production channels. We have shown that in the regions of parameter space where the Higgs boson searches at the LEP and Tevatron Colliders become difficult, the LHC will, in general, be able to find the Higgs $\phi_{W}$ boson at relatively low integrated luminosities, $\mathcal{L}<100 \mathrm{fb}^{-1}$. We have presented several explicit choices of MSSM parameters which demonstrate this point, including the possibility that the top and bottom squarks are relatively heavy or light. Interestingly enough, the $\phi_{W}$ couplings to bottom quarks and $\tau$ leptons, which control the dominant decay modes of the standard model Higgs boson, can be highly non-standard. A suppression of the standard signatures occurs for natural choices of the softsupersymmetry-breaking parameters.

In general, we have observed some patterns in the choices of soft, supersymmetry-breaking parameters that lead to difficulties at either LEP/Tevatron or LHC, but give a complementary enhanced signature at the other collider(s):

(i) When $A_{t} \mu<0$ or $A_{b} \mu>0$, with parameter values of the order of the scale $M_{S} \simeq 1 \mathrm{TeV}$, there can be a suppression of the $\phi_{W} b \bar{b}$ coupling, which limits the observability of the $\phi_{W} \rightarrow b \bar{b}$ channel at LEP/Tevatron. Complementary to this, $\operatorname{BR}\left(\phi_{W} \rightarrow \gamma \gamma\right)$ is enhanced at the LHC. Hence, while for these conditions the discovery of the Higgs $\phi_{W}$ boson at LEP and the Tevatron will require very high luminosities, even a low luminosity run at the LHC will be sufficient to discover the Higgs $\phi_{W}$ boson.

(ii) As the values of $A_{t}, A_{b}$ and $\mu$ are lowered, the $\phi_{W} b \bar{b}$ coupling can still be suppressed in the presence of large radiative corrections to the $\phi_{W} b \bar{b}$ coupling, $\Delta\left(m_{b}\right)$, which are proportional to $\tan \beta$ and can be of order one for large $\tan \beta$. In this case, the suppression occurs because the relation $\tan \alpha \simeq \Delta\left(m_{b}\right) / \tan \beta$ holds. There is also a mismatch between the $\phi_{W} b \bar{b}$ and $\phi_{W} \tau^{+} \tau^{-}$couplings, to be discussed below. As before, the complementarity arises because the LHC reach in the $\phi_{W} \longrightarrow \gamma \gamma$ channel increases (decreases) when the bottom Yukawa coupling is decreased (increased).

(iii) If $M_{S}$ is decreased, but the other parameters still obtain values near $1 \mathrm{TeV}$, then the $\phi_{W} b \bar{b}$ suppression occurs for smaller values of $\tan \beta$ and at large values of $m_{A}$. At large $\tan \beta$, the mass of the Higgs $\phi_{W}$ decreases from its upper bound, which is achieved at intermediate values of $\tan \beta$ between 10 and 20, and LEP becomes sensitive to the Higgs $\phi_{W}$ boson, but not in the regions where the Tevatron reach is also suppressed. The signal rate in the $\phi_{W} \rightarrow \gamma \gamma$ channel is again enhanced in those regions inaccessible at the LEP/Tevatron.

(iv) A small top squark mass, a large value for $A_{t}$ and moderate $\mu$ can decrease $\Gamma\left(\phi_{W} \rightarrow g g\right)$ through the interference of top and top squark loops, which limits the channel $g g \rightarrow \phi_{W} \rightarrow \gamma \gamma$ at the LHC. Simultaneously, the $\phi_{W} b \bar{b}$ coupling can be enhanced or suppressed over the SM value, because of the contribution of $A_{t}$ and $\mu$ to the mixing in the Higgs sector. If the $\phi_{W} b \bar{b}$ coupling is enhanced, the
$\mathrm{BR}\left(\phi_{W} \rightarrow \gamma \gamma\right)$ can actually also decrease. Because of the increase in $\operatorname{BR}\left(\phi_{W} \rightarrow b \bar{b}\right)$, the channel $\phi_{W} \rightarrow b \bar{b}$ at the Tevatron can be used to cover the problematic regions at the LHC, provided that the experiments at the Tevatron receive enough luminosity. On the other hand, if the $\phi_{W} b \bar{b}$ coupling is suppressed, there will be a further increase in $\operatorname{BR}\left(\phi_{W}\right.$ $\rightarrow \gamma \gamma$ ) which enhances the reach of the LHC in the $t \bar{t} \phi_{W}$ and $W \phi_{W}$ channels.

(v) Large values for $A_{b}, A_{t}$ and $\mu$ with light bottom and top squarks may lead to a wide suppression of $\operatorname{BR}\left(\phi_{W}\right.$ $\rightarrow \gamma \gamma$ ), because the $\phi_{W} b \bar{b}$ coupling can be significantly enhanced. This limits all of the $\phi_{W} \rightarrow \gamma \gamma$ channels at the LHC. The upper bound on the Higgs boson mass is reduced in conjunction with the increase in $\operatorname{BR}\left(\phi_{W} \rightarrow b \bar{b}\right)$, so that LEP and the Tevatron cover most of the complementary regions of the $M_{A}$ - $\tan \beta$ plane. High luminosity is only required at the Tevatron to cover the regions where neither $C P$-even Higgs boson has SM-like couplings to the gauge bosons, $\sin ^{2}(\beta-\alpha) \simeq \cos ^{2}(\beta-\alpha)$.

(vi) For MSSM parameter choices where the Higgs mixing would cause a suppression of both the $\phi_{W} b \bar{b}$ and $\phi_{W} \tau^{+} \tau^{-}$couplings at the tree level, large radiative corrections from SUSY-breaking effects can modify the bottom and tau decay rates in different ways. As a result, one may observe $\phi_{W}(\rightarrow b \bar{b})$ without $\phi_{W}\left(\rightarrow \tau^{+} \tau^{-}\right)$. In the regions where there is a suppression of the $\phi_{W} \tau^{+} \tau^{-}$coupling, there is not necessarily an enhancement of $\operatorname{BR}\left(\phi_{W} \rightarrow \gamma \gamma\right)$, because the $\phi_{W} b \bar{b}$ coupling can be of the order of the standard model value. In the presence of large SUSY-breaking effects, the suppression of the $\phi_{W} b \bar{b}$ coupling occurs when $\tan \alpha$ $\simeq \Delta\left(m_{b}\right) / \tan \beta$. For this value of $\tan \alpha$, the $\phi_{W} \tau^{+} \tau^{-}$coupling will not vanish, in general, and $\phi_{W} \rightarrow \tau^{+} \tau^{-}$may be the dominant decay.

While our analysis has emphasized the complementarity of LEP and the Tevatron in the $\phi_{W}(\rightarrow b \bar{b})$ channels to the LHC in the $\phi_{W}(\rightarrow \gamma \gamma)$ channels, our results are more general. If the experiments at the Tevatron do not receive enough luminosity, then the $t \bar{t} \phi_{W}(\rightarrow b \bar{b})$ channel, which to a good approximation has the same parameter dependence as the $W \phi_{W}(\rightarrow b \bar{b})$ channel, at the LHC will be complementary to the $\phi_{W}(\rightarrow \gamma \gamma)$ channel [the $W W \rightarrow \phi_{W}\left(\rightarrow \tau^{+} \tau^{-}\right)$ channel may also be useful]. On the other hand, with enough luminosity, the Tevatron may be able to observe the $\phi_{W}$ $(\rightarrow \gamma \gamma)$ channel when the $\phi_{W} b \bar{b}$ coupling is greatly suppressed.

\section{ACKNOWLEDGMENTS}

We thank D. Denegri and E. Richter-Was for providing information about various channels. We would also like to thank G. Azuelos, J. Gunion, H. Haber and J. Womersley for interesting discussions. M.C. and C.W. would like to thank the Aspen Center for Physics, where part of this work has been done. This work was supported in part by the U.S. Department of Energy, High Energy Physics Division, under Contract W-31-109-Eng-38. 


\section{APPENDIX: RELATIONS BETWEEN THE CP EVEN HIGGS BOSON MASSES}

To derive Eq. (1.2), we start with the Higgs boson squared-mass matrix elements parametrized in the following form (see Ref. [24]):

$$
\begin{aligned}
& \mathcal{M}_{11}^{2}=m_{A}^{2} \sin ^{2} \beta+\Delta_{11} \\
& \mathcal{M}_{22}^{2}=m_{A}^{2} \cos ^{2} \beta+\Delta_{22} \\
& \mathcal{M}_{12}^{2}=-m_{A}^{2} \cos \beta \sin \beta+\Delta_{12},
\end{aligned}
$$

where $\Delta_{i j}$ denotes terms independent of $m_{A}^{2}$. For very large values of $m_{A}^{2}$, the heaviest $C P$-even Higgs boson mass $m_{H}^{2}$ $\simeq m_{A}^{2}$ and the determinant of the Higgs boson squared mass matrix will be equal to $m_{A}^{2} \times\left. m_{h}^{2}\right|_{m_{A}^{2} \gg M_{Z}^{2}}$, where the last term is the upper bound on the lightest $C P$-even Higgs boson mass. This upper bound can be obtained by taking the terms proportional to $m_{A}^{2}$ in the determinant of the Higgs boson squared-mass matrix:

$$
\begin{aligned}
\left.m_{h}^{2}\right|_{m_{A}^{2} \gg M_{Z}^{2}} & \simeq \Delta_{11} \cos ^{2} \beta+\Delta_{22} \sin ^{2} \beta+2 \Delta_{12} \cos \beta \sin \beta \\
& =\mathcal{M}_{11}^{2} \cos ^{2} \beta+\mathcal{M}_{22}^{2} \sin ^{2} \beta+2 \mathcal{M}_{12}^{2} \cos \beta \sin \beta .
\end{aligned}
$$

Since the mass matrix is diagonalized by a rotation with mixing angle $\alpha$, we have

$$
\begin{aligned}
& \mathcal{M}_{11}^{2}=m_{h}^{2} \sin ^{2} \alpha+m_{H}^{2} \cos ^{2} \alpha \\
& \mathcal{M}_{22}^{2}=m_{h}^{2} \cos ^{2} \alpha+m_{H}^{2} \sin ^{2} \alpha \\
& \mathcal{M}_{12}^{2}=\left(m_{H}^{2}-m_{h}^{2}\right) \sin \alpha \cos \alpha .
\end{aligned}
$$

Substituting Eq. (A3) into Eq. (A2) yields the desired relation, namely,

$$
\left.m_{h}^{2}\right|_{m_{A}^{2} \gg M_{Z}^{2}}=m_{h}^{2} \sin ^{2}(\beta-\alpha)+m_{H}^{2} \cos ^{2}(\beta-\alpha) .
$$

Let us emphasize that, in the above, we have ignored the small differences between pole masses and running masses, while we have defined all matrix elements at the scale $m_{t}$, ignoring the effects of the decoupling of the heavy Higgs doublet. These effects, however, are only relevant for $m_{A}$ $\gg M_{Z}$, in which case $\sin ^{2}(\beta-\alpha) \rightarrow 1$ independently of the scale of definition. It is easy to prove that $\cos ^{2}(\beta-\alpha)$ $=\mathcal{O}\left(M_{Z}^{4} / m_{A}^{4}\right)$ for the same conditions, and therefore the above equality, Eq. (A4), is satisfied in a straightforward way.
[1] LEP Electroweak Working Group and SLD Heavy Flavor and Electroweak Groups: D. Abbaneo et al., Internal Note No. CERN-EP/99-15; J. Erler, hep-ph/9904235, and references therein.

[2] M. Carena, P. Zerwas and the Higgs Physics Working Group, in "Physics at LEP2," edited by G. Altarelli, T. Sjöstrand and F. Zwirner, CERN Report No. 96-01, Vol. 1.

[3] P. Dornan, talk presented at the LEPC meeting, CERN, 2000 (see http://alephwww.cern.ch); C. Mariotti, talk presented at the LEPC meeting, CERN, 2000 (see http:// delphiwww.cern.ch); M. Grunewald, talk presented at the LEPC meeting, CERN, 2000 (see http://13www.cern.ch); R. Mc Pherson, talk presented at the LEPC meetting, CERN, 2000 (see http://www.cern.ch/Opal).

[4] Y. Okada, M. Yamaguchi and T. Yanagida, Prog. Theor. Phys. 85, 1 (1991); H.E. Haber and R. Hempfling, Phys. Rev. Lett. 66, 1815 (1991); J. Ellis, G. Ridolfi and F. Zwirner, Phys. Lett. B 257, 83 (1991); 262, 477 (1991); A. Brignole, J. Ellis, G. Ridolfi and F. Zwirner, ibid. 271, 123 (1991).

[5] R. Hempfling and A. Hoang, Phys. Lett. B 331, 99 (1994); J. Kodaira, Y. Yasui and K. Sasaki, Phys. Rev. D 50, 7035 (1994)

[6] J. Casas, J.R. Espinosa, M. Quiros and A. Riotto, Nucl. Phys. B436, 3 (1995).

[7] M. Carena, J.-R. Espinosa, M. Quiros and C.E.M. Wagner, Phys. Lett. B 355, 209 (1995); M. Carena, M. Quiros and C.E.M. Wagner, Nucl. Phys. B461, 407 (1995).

[8] H. Haber, R. Hempfling and A.H. Hoang, Z. Phys. C 57, 539 (1997).
[9] S. Heinemeyer, W. Hollik and G. Weiglein, Phys. Rev. D 58, 091701 (1998); Eur. Phys. J. C 9, 343 (1999).

[10] See, for example, P. Chankowski, Proceedings of the International Workshop on Quantum Effects in the Minimal Supersymmetric Standard Model, Barcelona, Spain, 1997, pp. 87102; M. Carena, D. Choudhury, S. Raychaudhuri and C.E.M. Wagner, Phys. Lett. B 414, 414 (1997).

[11] M. Carena, P. Chankowski, S. Pokorski and C.E.M. Wagner, Phys. Lett. B 441, 205 (1998).

[12] J.R. Espinosa and D. Comelli, Phys. Lett. B 388, 793 (1996); J.R. Espinosa and J.F. Gunion, Phys. Rev. Lett. 82, 1084 (1999).

[13] M. Carena et al., Report of the Higgs Working Group of the RunII Workshop, Fermilab, 1999. Results available at http:// fnth37.fnal.gov/higgs.html

[14] D. Rainwater and D. Zeppenfeld, J. High Energy Phys. 12, 005 (1997); D. Rainwater, D. Zeppenfeld and K. Hagiwara, Phys. Rev. D 59, 014037 (1999); T. Plehn, D. Rainwater and D. Zeppenfeld, Phys. Lett. B 454, 297 (1999).

[15] G. Belanger, F. Boudjema and K. Sridhar, Nucl. Phys. B568, 3 (2000); A. Dedes and S. Moretti, Eur. Phys. J. C 10, 515 (1999).

[16] E. Richter-Was, D. Froidevaux, F. Gianotti, L. Poggioli, D. Cavalli and S. Resconi, Int. J. Mod. Phys. A 13, 1371 (1998).

[17] CMS Collaboration, G. Acquistapace et al., Report No. CERN-LHCC-97-10; R. Kinnunen and D. Denegri, Report No. CMS-NOTE-1997-057; K. Lassila-Perini, Report No. CMSTHESIS-1998-147.

[18] M. Carena, J. Ellis, S. Lola and C.E.M. Wagner, Eur. Phys. J. C 12, 507 (2000). 
[19] L. Hall, R. Rattazzi and U. Sarid, Phys. Rev. D 50, 7048 (1994); R. Hempfling, ibid. 49, 6168 (1994).

[20] M. Carena, M. Olechowski, S. Pokorski and C.E.M. Wagner, Nucl. Phys. B426, 269 (1994).

[21] D. Pierce, J. Bagger, K. Matchev and R. Zhang, Nucl. Phys. B491, 3 (1997).

[22] For related work see F. Borzumati, G.R. Farrar, N. Polonsky and S. Thomas, hep-ph/9712428; Nucl. Phys. B555, 53 (1999).

[23] J.A. Coarasa, R.A. Jimenez and J. Sola, Phys. Lett. B 389, 312 (1996); R.A. Jimenez and J. Sola, ibid. 389, 53 (1996).

[24] M. Carena, S. Mrenna and C.E.M. Wagner, Phys. Rev. D 60, 075010 (1999).

[25] K.S. Babu and C. Kolda, Phys. Lett. B 451, 77 (1998).
[26] A. Dabelstein, Nucl. Phys. B456, 25 (1995).

[27] See the transparencies from the talks of H. Logan and M.J. Herrero at SUSY99.

[28] H. Baer and J.D. Wells, Phys. Rev. D 57, 4446 (1998); W. Loinaz and J.D. Wells, Phys. Lett. B 445, 178 (1998).

[29] A. Djouadi, Phys. Lett. B 435, 101 (1998).

[30] As presented by P. Janot (CERN) at the Chamonix-IX Workshop, 1999.

[31] A. Djouadi, J. Kalinowski and M. Spira, Comput. Phys. Commun. 108, 56 (1998).

[32] H. Baer, B.W. Harris and X. Tata, Phys. Rev. D 59, 015003 (1999). 